\title{
Monte Carlo power iteration: entropy and spatial correlations
}

\author{
Michel Nowak ${ }^{\mathrm{a}}$, Jilang Miao ${ }^{\mathrm{b}}$, Eric Dumonteil ${ }^{\mathrm{a}, \mathrm{c}}$, Benoit Forget $^{\mathrm{b}}$, Anthony Onillon $^{\mathrm{c}}$, Kord S. Smith ${ }^{\mathrm{b}}$, Andrea Zoia $^{\mathrm{a}, *}$
}

${ }^{a}$ Den-Service d'études des réacteurs et de mathématiques appliquées (SERMA), CEA, Université Paris-Saclay, F-91191, Gif-sur-Yvette, France ${ }^{b}$ Department of Nuclear Science and Engineering, Massachusetts Institute of Technology, 77 Massachusetts Avenue, 24-107, Cambridge, MA 02139, United States ${ }^{c}$ IRSN, 31 Avenue de la Division Leclerc, 92260 Fontenay aux Roses, France

\begin{abstract}
The behaviour of Monte Carlo criticality simulations is often assessed by examining the convergence of the so-called entropy function. In this work, we shall show that the entropy function may lead to a misleading interpretation, and that potential issues occur when spatial correlations induced by fission events are important. We will support our analysis by examining the higher-order moments of the entropy function and the center of mass of the neutron population. Within the framework of a simplified model based on branching processes, we will relate the behaviour of the spatial fluctuations of the fission chains to the key parameters of the simulated system, namely, the number of particles per generation, the reactor size and the migration area. Numerical simulations of a fuel rod and of a whole core suggest that the obtained results are quite general and hold true also for real-world applications.
\end{abstract}

Keywords: Entropy, Clustering, Power iteration, OpenMC, TrIPOLI- $4^{\circledR}$

\section{Introduction}

Monte-Carlo simulation is often used in criticality calculations to assess the asymptotic distribution of the neutron population within a system, which corresponds to the fundamen5 tal eigenmode of the Boltzmann critical equation (Lux and Koblinger, 1991). The most widely used and simplest nu- ${ }^{35}$ merical method allowing the neutron population to converge to the fundamental eigenmode is the power iteration (Lux and Koblinger, 1991, Rief and Kschwendt, 1967, Brown, 2005): in

10 Monte Carlo methods, an initial arbitrary source particle distribution is transported until all neutrons have been either ab- ${ }^{40}$ sorbed or leaked (forming a so-called generation). The secondary neutrons coming from the fission events within a generation $g$ are banked and provide the source for the following 15 generation $g+1$. The algorithm is then iterated over many generations, until the fission sources for a sufficiently large $g$ statis- ${ }^{45}$ tically attain a spatial and energetic equilibrium, as ensured by the power iteration method. The effects of higher eigenmodes on the neutron population are expected to fade away, and even20 tually the neutron population will be distributed according to the fundamental eigenmode. The ratio between the population size at generation $g+1$ and the population size at generation $g$ converges to the fundamental eigenvalue $k_{\text {eff }}$ for large $g$ (Lux and Koblinger, 1991).

25 In this context, two key issues are known to affect the neutron population during power iteration and have therefore attracted intensive research efforts: fission source convergence and correlations.

\footnotetext{
${ }^{*}$ Corresponding author. Tel. +33 (0)1 69089544

Email addresses: jlmiao@mit.edu (Jilang Miao), andrea.zoia@cea.fr (Andrea Zoia)
}

Concerning the former, a slow exploration of the viable phase 30 space by the population implies a poor source convergence. In particular, it has been shown that the convergence of $k_{\text {eff }}$ might be faster than that of the associated fundamental eigenmode, which is expected on physical grounds, the former being an integral property of the system and the latter being a local property (Lux and Koblinger, 1991). The rate of convergence depends on the separation between the first and the second eigenvalue of the Boltzmann equation, the so-called dominance ratio: the closer to one the dominance ratio becomes, the poorer the convergence (Lux and Koblinger, 1991, Ueki et al. 2004; Dumonteil and Malvagi, 2012). If Monte-Carlo tallies are scored before attaining equilibrium, biases on the estimation of the variance may appear, and monitoring the convergence of $k_{\text {eff }}$ might be insufficient so as to determine the convergence of the whole population (Ueki and Brown, 2003, Dumonteil et al. 2006; LAbbate et al., 2007, Ueki, 2005).

Several tools have been proposed to assess the spatial convergence of fission sources, among which, one of the most popular, is the entropy of the fission sources (Ueki et al., 2003; Ueki. 2012, Ueki and Brown, 2003, Ueki, 2005). The idea behind the entropy function is to superimpose a regular Cartesian mesh to the viable space and to record the number of fission sites for each cell of the mesh, at each generation. This allows computing the so-called Shannon entropy $\mathcal{S}$ (Li and Vitany, 1997), which is defined as

$$
\mathcal{S}(g)=-\sum_{i, j, k} p_{i, j, k}(g) \log _{2}\left[p_{i, j, k}(g)\right],
$$

where $p_{i, j, k}(g)$ is the (statistically weighted) number of fission source particles in the cell of index $i, j, k$ at generation $g$ divided by the total number of source particles in all cells at generation $g$. The entropy function is expected to provide a measure of the 
phase space exploration as a function of the number of generations (Li and Vitany, 1997; Cover and Thomas, 1991): when the neutron distribution attains its stationary shape, the entropy $\mathcal{S}$ converges. A prominent advantage of the entropy is that $\mathcal{S}$ is a single scalar value whose evolution condenses the required 90 information on the spatial repartition. Moreover, as apparent from Eq. (1), the entropy $\mathcal{S}$ of the source distribution at generation $g$ is bounded, namely,

$$
0 \leq \mathcal{S}(g) \leq \log _{2} B
$$

where $B$ is the number of cells of the spatial mesh. This property ensures in particular that the variations of $\mathcal{S}$ will be bounded, and that the highest value of the entropy will be reached in the case of a perfect equipartition. The entropy func- ${ }_{100}$ on is nowadays a standard tool for most production Monte Carlo codes, although some concerns have been raised about possible issues related to its use in convergence diagnostics for loosely coupled multiplying systems (see, e.g., the analysis in (Shi and Petrovic, 2010a b; Ueki, 2005)).

55 The latter issue with power iteration in Monte Carlo simulation concerns the impact of correlations induced by fission events: physically speaking, a neutron can only be generated in the presence of a parent particle, which induces generationto-generation correlations (Lux and Koblinger, 1991; Sjenitzer 60 and Hoogenboom, 2011). This is a widely recognized problem, which is expected to affect the convergence of Monte Carlo scores and in particular make the applicability of Cen- ${ }^{110}$ tral Limit Theorem questionable (Ueki, 2012, Brown, 2009, Ueki, 2005). Correlations between generations have been of-

65 ten studied within the mathematical framework provided by the eigenvalue analysis of the Boltzmann critical equation (Brown. 2005, Sutton, 2014, 2015). Further work on correlations has concerned techniques aimed at improving the standard deviation estimates of Monte Carlo scores (Gelbard and Prael, 1990,

70 Ueki et al., 2003, 2004, Dumonteil and Malvagi, 2012, Ueki 2005). More recently, it has been pointed out that, due to the asymmetry between correlated births by fission and uncorrelated deaths by capture and leakage 11 neutrons initially prepared at equilibrium will be preferentially found clustered close

75 to each other after a few generations (Dumonteil et al., 2014). This peculiar phenomenon, named neutron clustering, might induce a strongly heterogeneous spatial repartition of the neutron population, which randomly evolves between generations ( $\mathrm{Du}-$ monteil et al., 2014; Zoia et al., 2014, de Mulatier et al., 2015).

80 The impact of neutron clustering has been determined to be inversely proportional to the number of neutrons per generation (Dumonteil et al. 2014).

In this paper, we will show that neutron clustering, not surprisingly, also affects the convergence of the fission sources: because of fission-induced correlations, the entropy function

\footnotetext{
${ }^{1}$ This phenomenon has been first investigated in the context of theoretical ${ }_{115}$ ecology, especially in relation to the evolution of biological communities: see, e.g., (Young et al. 2001. Houchmandzadeh 2008), and can be better understood in the framework of branching random walks (Athreya and Ney, 1972

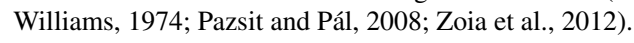

might in turn be ineffective at detecting potential deviations of the neutron population with respect to the expected equilibrium.

This manuscript is organized as follows. In Sec. 2. we will initially consider a simplified reactor model where exact analytical results can be established, and show that in some cases the convergence of the entropy is achieved, although the neutron population is still affected by strong spatial fluctuations. Then, in Sec. 3, we will refine our analysis based on the spatial moments of the entropy function and on the center of mass of the neutron population: these statistical tools can be used together with regular entropy so as to extract information concerning the simulated system, and thus improve the diagnosis of fission source equilibrium. In Sec. 4 we will then relate the behaviour of such spatial fluctuations to the key system parameters, namely, the reactor size, the number of neutrons per generation, and the migration area, based on the theory of branching processes. In Sec. 5] we will numerically explore the behaviour of a fuel rod and a full core with detailed geometry and compositions and continuous-energy treatment, and show that the theoretical findings for the simple reactor model actually apply more generally to realistic configurations. Conclusions will be drawn in Sec.6.

\section{Neutrons in a box and the behaviour of the entropy}

In order to assess the behaviour of the entropy function in the presence of fission-induced correlations, we will work out an example that is simple enough for exact results to be analytically derived and compared to the Monte Carlo simulations, and yet retains the key physical features of a real system (Miao et al. 2016).

Let us therefore consider a prototype model of a reactor core consisting of a collection of $N$ neutrons undergoing scattering, capture and fission within a box of volume $V=L^{3}$. To simplify the matter, we will assume that neutrons can only be reflected at the boundaries. The random displacements of the neutrons will be modelled by branching exponential flights with constant speed v; scattering and fission will be taken to be isotropic in the center of mass frame. The physical parameters of this prototype reactor will be the following:

$$
\Sigma_{s}=0.27, \quad \Sigma_{c}=0.02, \quad \Sigma_{f}=\frac{\Sigma_{c}}{\bar{v}-1.0}
$$

where $\Sigma_{s}$ is the scattering cross section, $\Sigma_{c}$ is the capture cross section, and $\Sigma_{f}$ is the fission cross section (in $\mathrm{cm}^{-1}$ ). The parameter $\bar{v}$ denotes the average number of secondary neutrons per fission. Observe that on the basis of the cross sections defined above the system is exactly critical, i.e.,

$$
k_{e f f}=\frac{\bar{v} \Sigma_{f}}{\Sigma_{c}+\Sigma_{f}}=1,
$$

for any choice of $\bar{v}$. For our simulations, we have set $\bar{v}=2.5$. The fundamental eigenmode associated to $k_{\text {eff }}=1$ corresponds to an equilibrium distribution that is spatially homogeneous over the box, as expected on physical grounds. 

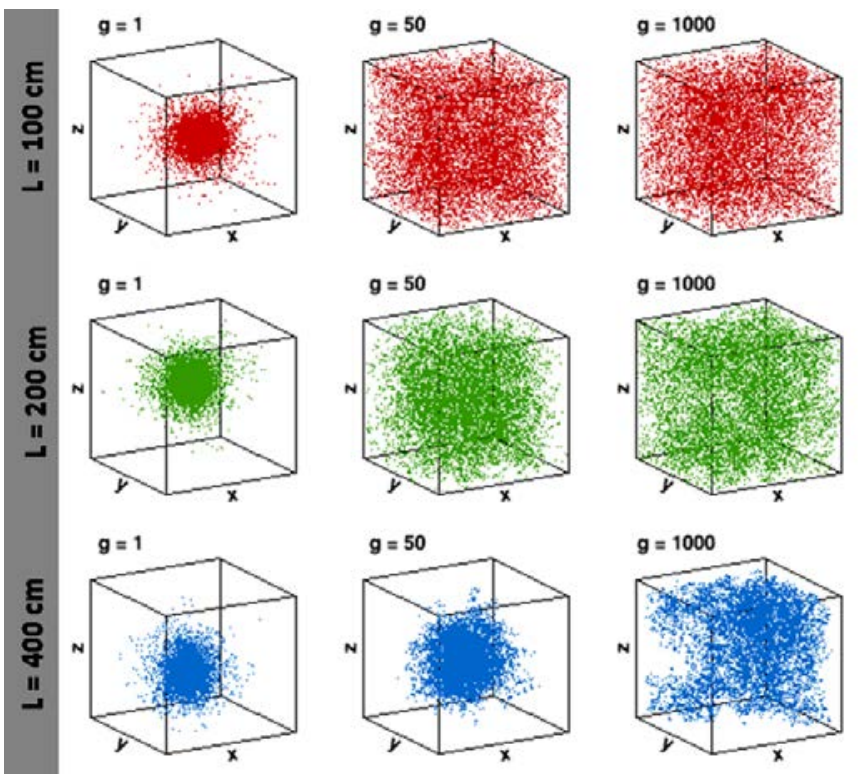

Figure 1: Distribution of fission sites during Monte Carlo power iteration as a function of generations $g$, for three different reactor sizes $L$. The guess source at $g=0$ consists of $N=10^{4}$ neutrons located at the center of the cube. Power iteration is run for 1000 generations. Top: $L=100 \mathrm{~cm}$; center: $L=200$ $\mathrm{cm}$; bottom: $L=400 \mathrm{~cm}$.

This prototype reactor model can be easily implemented and solved by power iteration within a Monte Carlo code. In order to probe the effects of neutron clustering on the convergence of power iteration, we have performed several Monte Carlo critical simulations of such system by varying the number $N$ of particles per generation and the size $L$ of the box, the other physical parameters being unchanged. For all configurations, we have assumed that the power iteration is started with a point source consisting of $N$ neutrons located at the center of the box. As the number of generation increases, the neutron population spreads over the whole box, and is forced to converge towards the fundamental eigenmode by the power iteration. However, this spread is counter-reacted by the spatial correlations induced by fission chains, and clustering might then come into play.

The distribution of the fission sites as obtained during the power iteration for a fixed number $N=10^{4}$ of initial particles different box sizes $L$ is displayed in Fig. 1. When the neutron density is high (i.e., $L$ is small for a given $N$ ), the fission sites converge to an equilibrium configuration where neutrons are homogeneously spread over the whole volume, with mild fluctuations mostly due to scattering. As $L$ increases, spatial the competing mechanisms of fission, absorption and scattering become more apparent. For even larger $L$, the neutron population displays patchiness, with neutrons randomly moving around the box grouped into a large cluster. Previous investigations based on the diffusion theory approximation have indeed shown that spatial clustering is quenched when $L^{2} \ll N \mathcal{M}^{2}$, where $\mathcal{M}^{2}$ is the migration area (de Mulatier et al., 2015). Fluctuations due to correlations are therefore ex-150

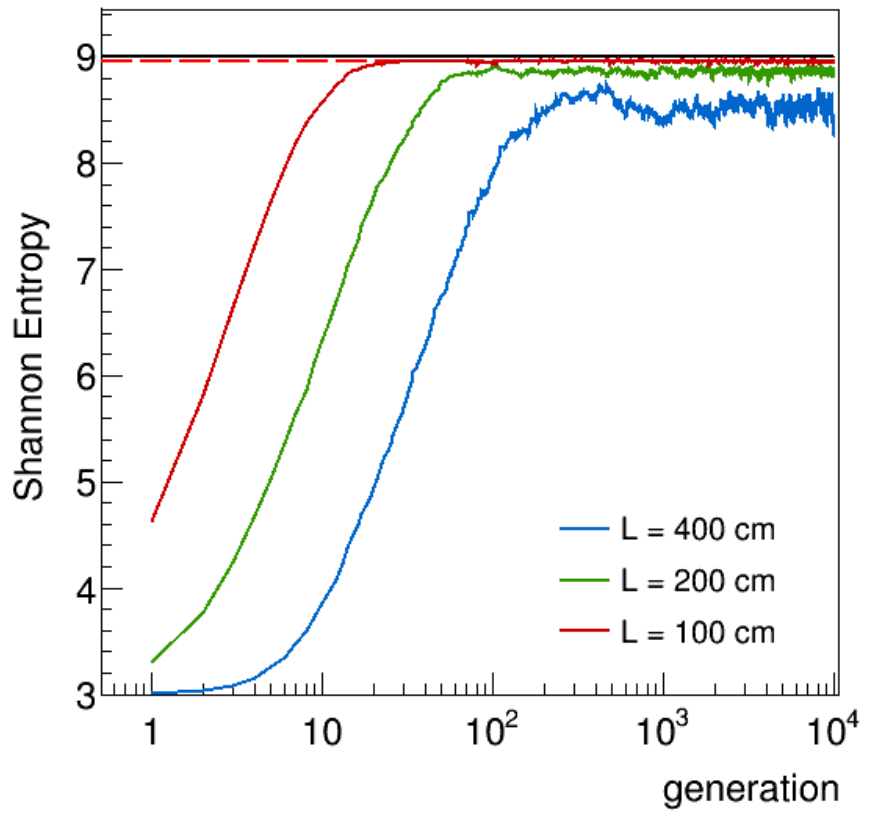

Figure 2: Homogeneous cube reactor. The behaviour of the measured Shannon entropy $\mathcal{S}(g)$ during Monte Carlo power iteration as a function of generations $g$, for three different reactor sizes $L$ and fixed number $N$ of neutrons per generation. The guess source at $g=0$ consists of $N=10^{4}$ neutrons located at the center of the cube. Power iteration is run for 1000 generations. Upper red curve: $L=100 \mathrm{~cm}$; central green curve: $L=200 \mathrm{~cm}$; lower blue curve: $L=400 \mathrm{~cm}$. The dashed red line represents the expected entropy value $\mathcal{S}_{N}$ as in Eq. [6, and the solid black line is the ideal expected entropy $\mathcal{S}_{\infty}$ for an infinite number of particles per generation as in Eq. (5).

pected to decrease for decreasing box size $L$ when keeping $N$ constant, which is coherent with our numerical findings.

It is instructive to compute the Shannon entropy $\mathcal{S}(g)$ for the power iteration simulations examined here. To fix the ideas, we will assume that each box side $L$ is partitioned into 8 spatial meshes, which implies $B=8^{3}=512$. For the simple model considered in this Section, the theoretical ideal entropy associated to the fundamental eigenmode, i.e., the expectation of $\log (p)$ corresponding to a uniform spatial distribution, can be exactly computed, and reads

$$
\mathcal{S}_{\infty}=\log _{2}(B)=9 .
$$

Actually, for a finite number $N$ of particles per generation, the theoretical expected Shannon entropy is always lower than the ideal value $\mathcal{S}_{\infty}$. For the homogeneous reactor, the expected Shannon entropy at finite $N$ can be explicitly computed (derivation is provided in Appendix A and reads

$$
\mathcal{S}_{N}=\log _{2}(N)-\frac{B^{1-N}}{N} \sum_{k=0}^{N}\left(\begin{array}{l}
N \\
k
\end{array}\right)(B-1)^{N-k} k \log _{2}(k),
$$

which depends on the number of spatial meshes $B$ and of the 

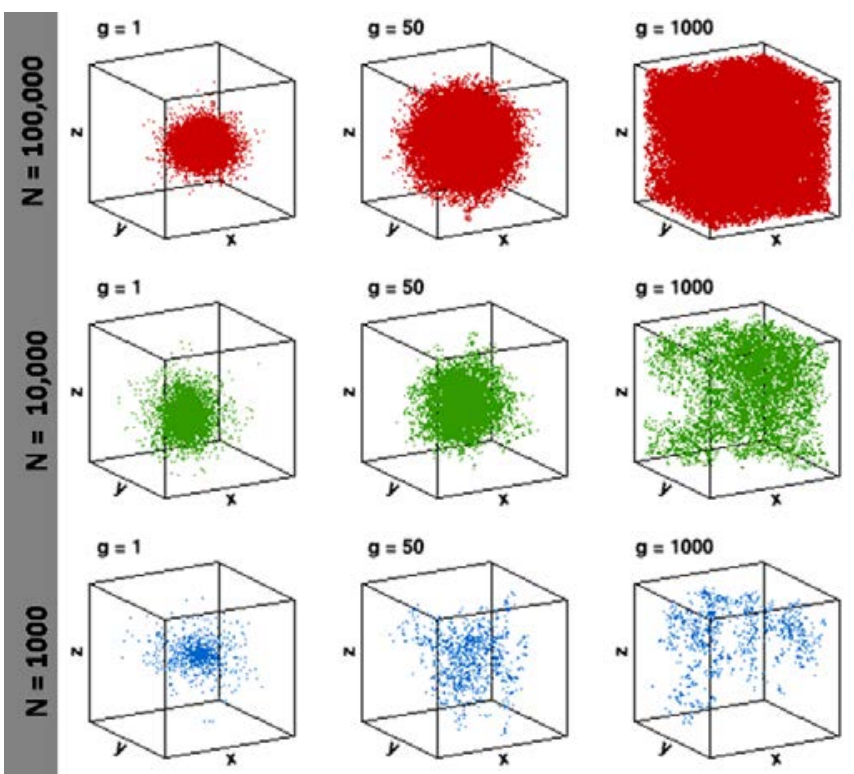

Figure 3: Homogeneous cube reactor. Distribution of fission sites during Monte Carlo power iteration as a function of generations $g$, for three different initial population sizes $N$ and fixed $L=400 \mathrm{~cm}$. The guess source at $g=0$ consists of $N$ neutrons located at the center of the cube. Power iteration is run for 1000 generations. Top: $N=10^{5}$; center: $N=10^{4}$; bottom: $N=10^{3}$.

number of particles $N$. It can be shown that $\mathcal{S}_{N}<\mathcal{S}_{\infty}$; when the number of neutrons $N$ is very large, the expected entropy converges to the ideal reference value, namely, $\mathcal{S}_{N} \rightarrow \mathcal{S}_{\infty}$. In the following, we will compare the measured entropy $\mathcal{S}(g)$ as a

function of generations to the expected value $\mathcal{S}_{N}$.

The behaviour of the measured entropy $\mathcal{S}(g)$ corresponding to the reactor configurations presented in Fig. 1 is displayed in Fig. 2. The number $m$ of generations taken by the neutron population to achieve spatial convergence (i.e., to explore the reactor) starting from a point source can be roughly estimated by $m \simeq L^{2} / \ell^{2}, \ell^{2}$ being the mean square displacement of a particle per generation. The quantity $\ell^{2}$ can be estimated during the Monte Carlo simulation, and for the example discussed here we have $\ell^{2} \simeq 175 \mathrm{~cm}^{2}$ for $L=100 \mathrm{~cm}, \ell^{2} \simeq 185 \mathrm{~cm}^{2}$ for $m \simeq 57$ for $L=100 \mathrm{~cm}, m \simeq 215$ for $L=200 \mathrm{~cm}$, and $m \simeq 830$ for $L=400 \mathrm{~cm}$, respectively. This is consistent with the number of generations taken by the measured entropy $\mathcal{S}(g)$ to attain convergence, as shown in Fig. 2 .

When $L=100 \mathrm{~cm}$, the computed $\mathcal{S}(g)$ asymptotically con-190 verges to the expected value $\mathcal{S}_{N}$ for large $g$ : in this case, the entropy function correctly mirrors the equilibrium attained by the neutron population. As $L$ increases by keeping $N$ fixed, spatial clustering strongly affects the neutron population during the power iteration: the neutron population still attains $a_{195}$ stationary equilibrium distribution, which is nonetheless quite different from the flat fundamental eigenmode. In particular, a larger fraction of empty cells is observed. The measured entropy $\mathcal{S}(g)$ consequently converges to an asymptotic value for

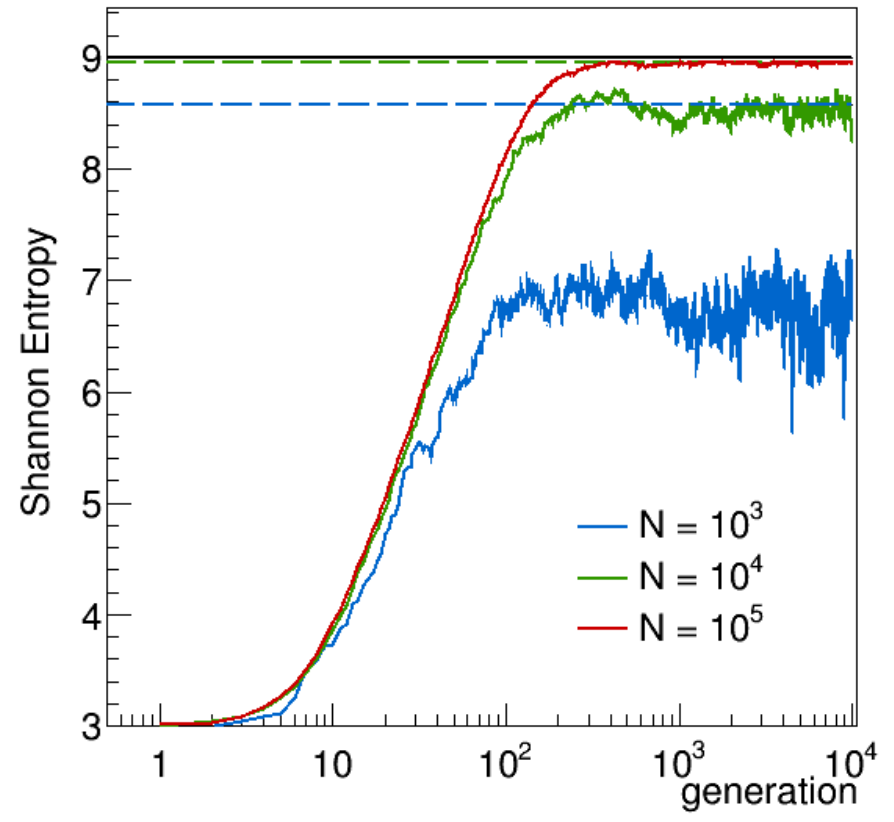

Figure 4: Homogeneous cube reactor. The behaviour of the measured Shannon entropy $\mathcal{S}(g)$ during Monte Carlo power iteration as a function of generations $g$, for different initial population sizes $N$ and fixed $L=400 \mathrm{~cm}$. The guess source at $g=0$ consists of $N$ neutrons located at the center of the cube. Power iteration is run for 1000 generations. Upper red curve: $N=10^{5}$; central green curve: $N=10^{4}$; lower blue curve: $N=10^{3}$. The dashed lines represent the expected entropy value $\mathcal{S}_{N}$ as in Eq. (6) (red: $N=10^{5}$, green: $N=10^{4}$ and blue: $N=10^{3}$, respectively), and the solid black line is the ideal expected entropy $\mathcal{S}_{\infty}$ for an infinite number of particles per generation as in Eq. (5).

large $g$ which is lower than the expected $\mathcal{S}_{N}$ and decreases with increasing $L$. Numerical analysis shows that the ratio between the measured and the asymptotic value of the Shannon entropy scales as $\left(\mathcal{S}_{N}-\mathcal{S}(g)\right) / \mathcal{S}_{N} \propto 1 / L$ for fixed $N$.

For the homogeneous reactor examined here, where $\mathcal{S}_{N}$ can be exactly determined, measuring the discrepancy between $\mathcal{S}(g)$ and $\mathcal{S}_{N}$ at convergence allows in principle the anomalous behaviour of the fission source convergence to be detected. In general, however, it is not possible to compute the expected entropy value $\mathcal{S}_{N}$, which means that in the presence of strong spatial clustering assessing the convergence of the Shannon entropy may turn out to be insufficient to ensure a proper spatial convergence of the fission sources.

We have carried a similar analysis for the power iteration by varying the number $N$ of initial neutrons per generation at fixed reactor size $L$. The results are displayed in Figure 3 The pattern followed by the neutron population during convergence is similar to that discussed in the analysis carried out above. When the neutron density is high (i.e., $N$ is large for a given $L$ ), the fission sites converge to a spatial equilibrium with neutrons 

fluctuations mostly due to scattering. As $N$ decreases, spatial fluctuations become more apparent, and for even smaller populations neutron clustering eventually sets in. On the basis of the argument discussed above, fluctuations due to correlations 205 are expected to increase for decreasing population size $N$ when keeping $L$ constant, which is coherent with our numerical findings.

The behaviour of the measured Shannon entropy $\mathcal{S}(g)$ for the configurations presented in Fig. 3 is displayed in Fig. 4 The expected entropy value $\mathcal{S}_{N}$ depends on the number of particles per generation, whereas the ideal asymptotic value $\mathcal{S}_{\infty}$ is unchanged and reads $\mathcal{S}_{\infty}=9$. The number $m$ of generations taken by the neutron population to achieve spatial convergence starting from the point source is again $m \simeq L^{2} / \ell^{2}$, with $\ell^{2} \simeq 190$ $m^{2}$ and $L=400 \mathrm{~cm}$, which yields $m \simeq 830$, independently of the number of simulated neutrons. This is consistent with the number of generations taken by the measured entropy $\mathcal{S}(g)$ to attain convergence, as shown in Fig. 4

When the number of initial neutrons $N$ is sufficiently large, the Shannon entropy converges to an asymptotic value that is very close to $\mathcal{S}_{N}$. As the relevance of the spatial clustering increases for decreasing $N$, the fraction of empty cells at equilibrium increases, and the asymptotic value of $\mathcal{S}(g)$ attained at convergence becomes progressively lower than $\mathcal{S}_{N}$. Numerical analysis shows that the ratio between the measured and expected entropy scales as $\left(\mathcal{S}_{N}-\mathcal{S}(g)\right) / \mathcal{S}_{N} \propto 1 / N$. Similarly as observed above, the Shannon entropy may thus become ineffective in diagnosing fission source convergence in the presence of spatial clustering.

One might wonder whether the results discussed in this Section are specific to exactly critical configurations (i.e., to having chosen $k_{\text {eff }}=1$ ). Actually, this is not the case. We have performed several other Monte Carlo power iteration simulations for super- or sub-critical reactors by varying the system 255 parameters, and the outcomes are qualitatively similar to those presented here. Finally, the behaviour of the entropy function with respect to the mesh number $B$ is presented in Fig. 5. The convergence to the asymptotic value $\mathcal{S}_{N}$ for any fixed $N$ decreases for increasing $B$, which is clearly understood on physthe number of particles $N$ required to smooth out the effects of correlations in each spatial bin must be also larger. In particular, we have numerically observed that we have the scaling $\left(\mathcal{S}_{N}-\mathcal{S}(g)\right) / \mathcal{S}_{N} \propto B^{1 / 3}$ for fixed $N$ and $L$.

\section{Beyond entropy: extracting information from spatial moments}

The main drawback of the entropy function as a tool for the statistical analysis of Monte Carlo power iteration is that the 265 spatial fluctuations of the neutron population are somehow averaged out by the sum over all cells in Eq.1] an asymptotic convergence can be ultimately attained even if neutrons are subject to strong (but statistically stationary in space) patchiness. Clustering effects will then go undetected when using the standard 270 definition of $\mathcal{S}(g)$ during power iteration, unless the theoretical

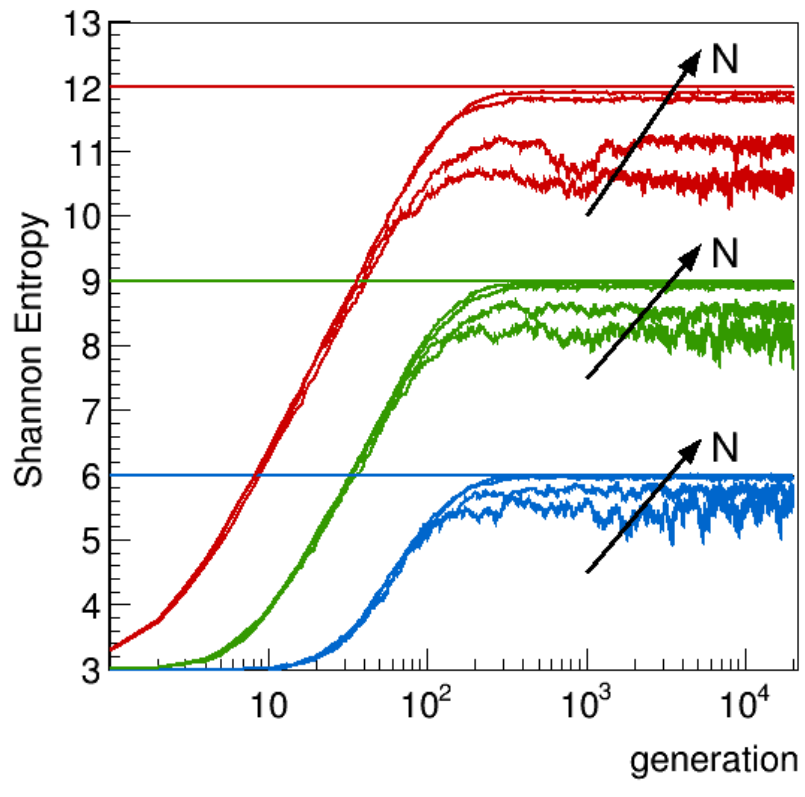

Figure 5: Homogeneous cube reactor. The behaviour of the measured Shannon entropy $\mathcal{S}(g)$ during Monte Carlo power iteration as a function of generations $g$, for different mesh number $B$, and fixed $L=400 \mathrm{~cm}$. The guess source at $g=0$ consists of $N$ neutrons located at the center of the cube. Power iteration is run for $2 \times 10^{4}$ generations. Upper red curves: $B=16 \times 16 \times 16$; central green curves: $B=8 \times 8 \times 8$; lower blue curve: $B=4 \times 4 \times 4$. For any fixed $B, N$ is progressively increased, i.e., $N=5 \times 10^{3}, N=10^{4}, N=5 \times 10^{4}, N=10^{5}$. For reference, the corresponding theoretical entropy values $\mathcal{S}_{\infty}$ are displayed as solid lines.

value $\mathcal{S}_{N}$ is known in advance, so that the ratio $\mathcal{S}(g) / \mathcal{S}_{N}$ can be computed. Generally speaking, this is however not possible but for the simplest reactor configurations, such as the homogeneous cube considered above.

In order to explicitly include the effects of spatial correlations in our statistical analysis, a convenient choice consists in generalizing the entropy function defined in Eq. 1 as follows:

$$
\mathcal{S}_{u, v, w}^{*}=-\sum_{i, j, k} \mathcal{L}_{u}\left(\xi_{i}\right) \mathcal{L}_{v}\left(\xi_{j}\right) \mathcal{L}_{w}\left(\xi_{k}\right) p_{i, j, k} \log _{2}\left[p_{i, j, k}\right],
$$

where $\mathcal{L}_{q}(\zeta)$ are the Legendre polynomials (other basis sets could also be used) of order $q$ and argument $\zeta$, and $\xi_{i}$ is the $x$-coordinate $\left(\xi_{j}\right.$ is the $y$-coordinate, and $\xi_{k}$ is the $z$-coordinate, respectively) of the center of the cell $i, j, k$, normalized to the interval $[-1,1]$. When $u=v=w=0$, we recover the regular Shannon entropy function, namely, $\mathcal{S}_{0,0,0}^{*}(g)=\mathcal{S}(g)$. For $q=1$, we have $\mathcal{L}_{1}(\zeta)=\zeta$, so that the quantity $\mathcal{S}_{1,0,0}^{*}(g)$ can be interpreted as the first spatial moment of the entropy function along the $x$ direction $\left(\mathcal{S}_{0,1,0}^{*}(g)\right.$ in the $y$ direction and $\mathcal{S}_{0,0,1}^{*}(g)$ in the $z$ direction, respectively).

By construction, the generalized entropy function $\mathcal{S}_{u, v, w}^{*}(g)$ is better suited than $\mathcal{S}(g)$ in detecting spatial fluctuations during the Monte Carlo power iteration. In order to illustrate our 

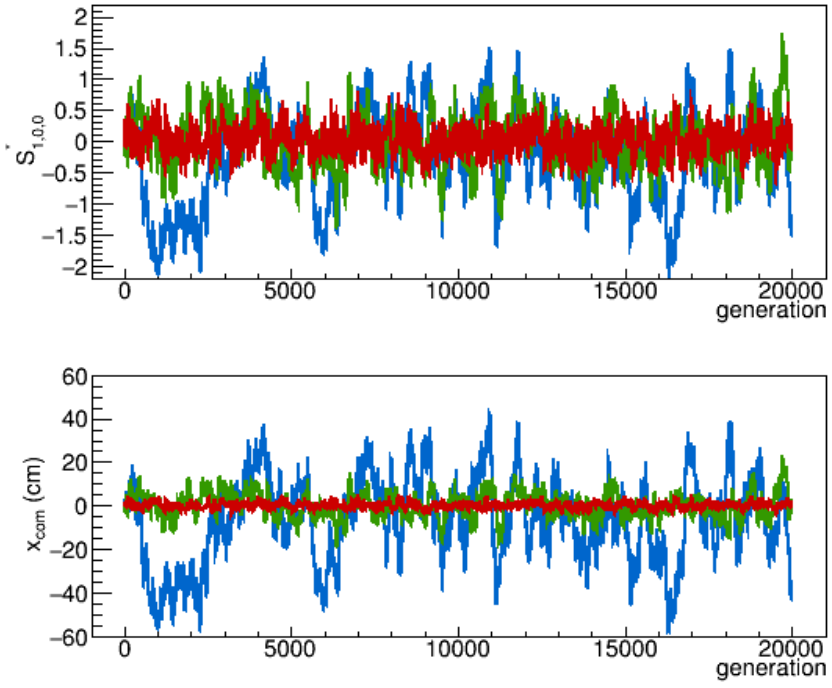

Figure 6: Homogeneous cube reactor. Monte Carlo power iteration as a function of generations $g$, for three different reactor sizes $L$ and fixed number $N$ of neutrons per generation. The guess source at $g=0$ consists of $N=10^{4}$ neutrons located at the center of the cube. Power iteration is run for 1000 generations. Top. The behaviour of the generalized Shannon entropy $\mathcal{S}_{1,0,0}^{*}(g)$. Red curve: $L=100 \mathrm{~cm}$; Green curve: $L=200 \mathrm{~cm}$; Blue curve: $L=400 \mathrm{~cm}$. Bottom. The behaviour of the center of mass $x_{\text {com }}(\mathrm{g})$. Red curve: $L=100 \mathrm{~cm}$; Green curve: $L=200$ cm; Blue curve: $L=400 \mathrm{~cm}$.

argument, let us revisit the homogeneous cubic reactor of the previous Section, with a fixed number of meshes $B=512$. The behaviour of $\mathcal{S}_{1,0,0}^{*}(g)$ for the reactor configurations obtained by keeping the number of neutrons $N$ fixed and varying the box size $L$ is illustrated in Fig. 6 (top). The first spatial moment of the entropy along the $x$ direction is expected to be zero due to the symmetry of neutron distribution. Since the source for the power iteration is initially placed at the center of the box, there is no appreciable convergence phase for $\mathcal{S}_{1,0,0}^{*}(g)$, as opposed to $\mathcal{S}(g)$. Fluctuations of $\mathcal{S}_{1,0,0}^{*}(g)$ around zero clearly mirror the spatial correlations: as $L$ increases, correlations become stronger and the fluctuations of $\mathcal{S}_{1,0,0}^{*}(g)$ become wilder. A similar behaviour is found when decreasing the number $N$ of ${ }_{295}$ simulated neutrons per generations at fixed reactor size $L$, as shown in Fig. 7 (top). When $N$ is large, the $\mathcal{S}_{1,0,0}^{*}(g)$ is again close to zero, and the fluctuations increase by decreasing $N$. For symmetry reasons, the results for $\mathcal{S}_{0,1,0}^{*}(g)$ and $\mathcal{S}_{0,0,1}^{*}(g)$ are (statistically) identical to those obtained for $\mathcal{S}_{1,0,0}^{*}(g)$ and will ${ }_{300}$ thus not be shown here.

A second, and perhaps more intuitive, approach to the analysis of spatial fluctuations during power iteration consists in computing the center of mass $\boldsymbol{r}_{\text {com }}(g)$ of the neutrons as a function of generations $g$, as recently suggested for instance 305 by (Wenner and Haghighat, 2007, 2008). To be more precise, for a collection of $N$ particles having coordinates $\left\{x_{1}, y_{1}, z_{1}, \cdots\right.$ $\left.x_{i}, y_{i}, z_{i}, \cdots x_{N}, y_{N}, z_{N}\right\}$ at the fission sites, the center of mass is
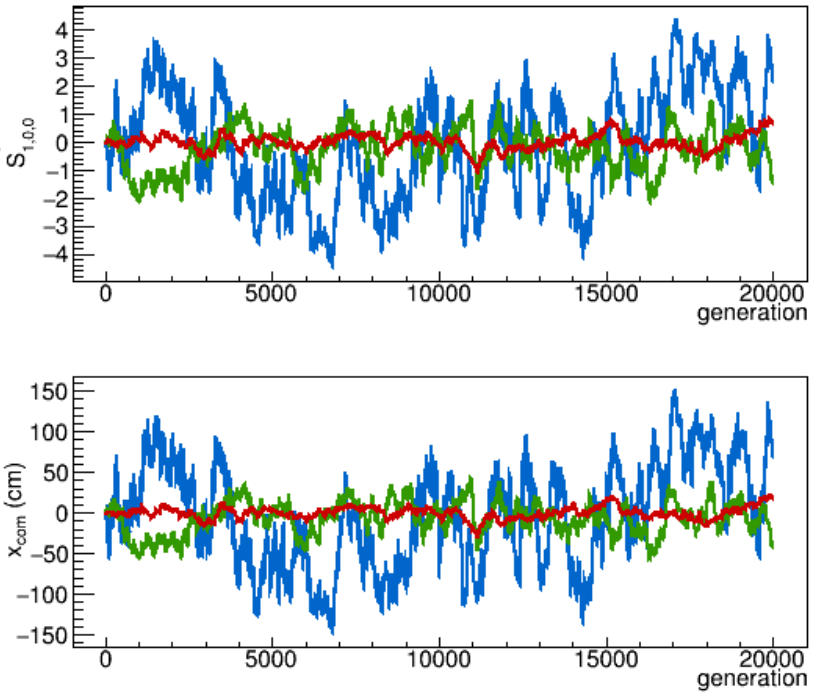

Figure 7: Homogeneous cube reactor. Monte Carlo power iteration as a function of generations $g$, for three different initial population sizes $N$ and fixed $L=400 \mathrm{~cm}$. The guess source at $g=0$ consists of $N$ neutrons located at the center of the cube. Power iteration is run for 1000 generations. Top. The behaviour of the generalized Shannon entropy $\mathcal{S}_{1,0,0}^{*}(g)$. Red curve: $N=10^{5}$; green curve: $N=10^{4}$; blue curve: $N=10^{3}$. Bottom. The behaviour of the center of mass $x_{\text {com }}(g)$. Red curve: $N=10^{5}$; green curve: $N=10^{4}$; blue curve: $N=10^{3}$.

defined as

$$
\mathbf{r}_{c o m}(g)=\frac{\sum_{i} w_{i} \mathbf{r}_{i}}{\sum_{i} w_{i}},
$$

where $\mathbf{r}_{i}$ is the vector of components $\mathbf{r}_{i}=\left\{x_{i}, y_{i}, z_{i}\right\}$, and $w_{i}$ are the statistical weights of the neutrons. The components of the center of mass along each direction are defined as

$$
x_{\text {com }}(g)=\frac{\sum_{i} w_{i} x_{i}}{\sum_{i} w_{i}}, y_{\text {com }}(g)=\frac{\sum_{i} w_{i} y_{i}}{\sum_{i} w_{i}}, z_{\text {com }}(g)=\frac{\sum_{i} w_{i} z_{i}}{\sum_{i} w_{i}} .
$$

Observe that by construction the center of mass $\mathbf{r}_{\text {com }}(g)$ does not depend on the number of spatial meshes.

The analysis of the center of mass estimator for the reactor configurations obtained by keeping the number of neutrons $N$ fixed and varying the box size $L$ is illustrated in Fig. 6 (bottom), where we display $x_{\text {com }}(g)$. Since the source for the power iteration is initially placed at the center of the box, there is no appreciable convergence phase for $x_{\text {com }}(g)$. The evolution of $x_{\text {com }}(g)$ clearly mirrors the spatial fluctuations of the neutron population: when $L$ is small, and the population is uniformly distributed within the box, $x_{\text {com }}(g)$ fluctuates around the symmetry center (here, $x=y=z=0$ ), and the fluctuations are rather mild. As $L$ increases, the effects of spatial correlations becomes stronger, and the evolution of $x_{\text {com }}(g)$ becomes increasingly erratic. The qualitative behaviour of $x_{\text {com }}(g)$ is closely related to that of $\mathcal{S}_{1,0,0}^{*}(g)$, apart from the normalizing factor that is imposed by construction in the Legendre polynomials for $\mathcal{S}_{u, v, w}^{*}(g)$. 
A similar behaviour is again found for $x_{\text {com }}(g)$ when decreasreactor size $L$, as shown in Fig. 7 (bottom). The fluctuations 360 of $x_{\text {com }}(g)$ increase by decreasing $N$, and the qualitative evolution of $x_{\text {com }}(g)$ is strikingly similar to that of $\mathcal{S}_{1,0,0}^{*}(g)$. For symmetry reasons, the results for $y_{\text {com }}(g)$ and $z_{\text {com }}(g)$ are (stanot be shown here.

\section{The impact of clustering on the spatial moments}

A deeper understanding of the qualitative behaviour of the generalized entropy and by the center of mass that we have observed in the previous Section can be achieved by relating the features of these estimators to the key physical parameters that govern the evolution of the Monte Carlo power iteration. This is actually possible by resorting to the theory of branching stochastic processes. Since the features of the generalized enare almost identical to those of the center of mass, for our analysis in the following we will focus on this latter.

The nuclear reactor model described above can be conceptually represented as a collection of $N$ particles undergoing scattering, reproduction and absorption within a homogeneous box of finite volume $V$, with reflecting (mass-preserving) boundaries. In order to keep notation simple, and yet retain the key ingredients of the model, we will approximate the exponential paths of the neutrons by regular Brownian motion with a constant diffusion coefficient $D$ (in other words, we are assuming that the diffusion approximation holds). For the same reason, instead of working with discrete generations we will introduce a continuous time $t$. The diffusing walker undergoes a birth-death event at rate $\beta=\mathrm{v} \Sigma_{f}$ : the neutron disappears and is replaced by a random number $k$ of descendants, distributed according to a law $q_{k}$ with average $\bar{v}_{f}=\sum_{k} k q_{k}$. We will assume that exactly two neutrons are emitted at fission.

In the Monte Carlo power iteration, some population control mechanisms are typically applied (such as Russian roulette and splitting), and the neutron population is typically normalized at ${ }^{370}$ the end of each generation in order to prevent the number of individuals from either exploding or shrinking to zero. For our aims, the effect of such population control mechanisms on our model can be mimicked by imposing that the total number $N$ of neutrons in $V$ is preserved. The simplest way to ensure a constant $N$ is to correlate fission and capture events (Zhang et al. 1990, Meyer et al. 1996): at each fission, a neutron disappears and is replaced by 2 descendants, and 1 neutron is simultaneously captured (i.e., removed from the collection) in order to ensure the conservation of total population ${ }^{2}$.

Analysis of this model shows that the evolution of the neutron population is governed by two distinct time scales: a mixing time $\tau_{D} \propto V^{2 / 3} / D$ and a renewal time $\tau_{R} \propto N / \beta$. The

${ }^{2}$ This mechanism has been first introduced in the theoretical ecology [Zhang et al. 1990 Meyer et al. 1996), where similar large-scale constraints have been shown to quench the wild fluctuations in the number of individuals that are expected for an unconstrained community. quantity $\tau_{D}$ physically represents the time over which a particle has explored the finite viable volume $V$ by diffusion. The emergence of the time scale $\tau_{D}$ is a distinct feature of confined geometries having a finite spatial size: for unbounded domains, $\tau_{D} \rightarrow \infty$. The quantity $\tau_{R}$ represents the time over which the system has undergone a population renewal, and all the individuals descend from a single common ancestor. When the concentration $N / V$ of individuals in the population is large (and the system is spatially bounded), it is reasonable to assume that $\tau_{R}>\tau_{D}$.

Consider then a collection of $N$ such particles moving around randomly, whose vector positions $\mathbf{r}_{i}(t), i=1, \cdots, N$, are recorded at time $t$. The spatial behaviour of the individuals can be characterized in terms of several moments, namely, the square center of mass

$$
\left\langle r_{\text {com }}^{2}\right\rangle(t) \equiv\left\langle\left|\frac{1}{N} \sum_{i} \mathbf{r}_{i}(t)\right|^{2}\right\rangle,
$$

the mean square displacement

$$
\left\langle r^{2}\right\rangle(t) \equiv \frac{1}{N} \sum_{i}\left\langle\left|\mathbf{r}_{i}(t)\right|^{2}\right\rangle,
$$

and the mean square distance between pairs of particles

$$
\left\langle r_{p}^{2}\right\rangle(t) \equiv \frac{1}{N(N-1)} \sum_{i, j}\left\langle\left|\mathbf{r}_{i}(t)-\mathbf{r}_{j}(t)\right|^{2}\right\rangle .
$$

Brackets denote the expectation with respect to the ensemble of possible realizations.

By construction, these three quantities are related to each other. By developing the series in the definitions above, we can in particular express the center of mass as a function of the mean square displacement and of the particle pair distance (Meyer et al. 1996), namely,

$$
\left\langle r_{\text {com }}^{2}\right\rangle(t)=\left\langle r^{2}\right\rangle(t)-\frac{1}{2} \frac{N-1}{N}\left\langle r_{p}^{2}\right\rangle(t)
$$

In the following, we will explicitly compute the center of mass for the homogeneous reactor and relate its behaviour to the model parameters.

\subsection{Neutron density and correlations}

Let us denote by $n(\mathbf{x}, t)$ the instantaneous density of neutrons located at $\mathbf{x}$ at time $t$. For a critical reactor, the average neutron density at a point $\mathbf{x}$ reads

$$
\langle n(\mathbf{x}, t)\rangle=N \rho(\mathbf{x}, t),
$$

where we have set

$$
\rho(\mathbf{x}, t)=\int d \mathbf{x}_{0} Q\left(\mathbf{x}_{0}\right) \mathcal{G}\left(\mathbf{x}, \mathbf{x}_{0}, t\right) .
$$

Here $Q$ is the spatial probability distribution function of the neutrons at time $t=0$, and the Green's function $\mathcal{G}\left(\mathbf{x}, \mathbf{x}_{0}, t\right)$ satisfies the backward diffusion equation

$$
\frac{\partial}{\partial t} \mathcal{G}\left(\mathbf{x}, \mathbf{x}_{0}, t\right)=D \nabla_{\mathbf{x}_{0}}^{2} \mathcal{G}\left(\mathbf{x}, \mathbf{x}_{0}, t\right),
$$


with the appropriate boundary conditions de Mulatier et al. 375

- Assuming that the initial neutron population has a uniform spatial distribution, we have $Q=1 / V$, and the average neutron density at any time will be spatially uniform, namely, $\langle n(\mathbf{x}, t)\rangle=N / V$. For arbitrarily distributed sources at $t=0$, the neutron density will asymptotically converge towards $\langle n(\mathbf{x}, t \rightarrow \infty)\rangle=N / V$ for long times.

By construction, the average density does not convey any information concerning the spatial fluctuations of the neutron population. In order to go beyond the average behaviour, we introduce the pair correlation function $h(\mathbf{x}, \mathbf{y}, t)$ between positions $\mathbf{x}$ and $\mathbf{y}$, which is proportional to the probability density of finding a pair of particles with the former at $\mathbf{x}$ and the latter at $\mathbf{y}$ (de Mulatier et al. 2015). It is important to stress that the correlation length can be extracted from the shape of the function $h$ : if $h$ is almost flat in space, then the correlations will have the same relevance at any spatial site; on the contrary, the presence of a peak in $h$ at short distances might reveal clustering phenomena (Zhang et al., 1990, Meyer et al., 1996). The overall intensity of the correlations is simply provided by the amplitude of $h$. When the particles can only diffuse (i.e., if the probability of fission and capture are artificially suppressed), the neutron population behave as an ideal gas of $N$ independent random walkers, and the corresponding correlation function yields

$$
h_{i d}(\mathbf{x}, \mathbf{y}, t)=N(N-1) \rho(\mathbf{x}, t) \rho(\mathbf{y}, t) .
$$

For the particular configuration where the particles are uniformly distributed at the initial time, $h_{i d}(\mathbf{x}, \mathbf{y}, t)=N(N-1) / V^{2}$.

For the homogeneous reactor model defined above, the pair correlation function $h$ can be exactly computed by resorting to the approach originally proposed in (Meyer et al., 1996) for infinite domains and later refined by (de Mulatier et al. 2015) for bounded domains. The calculations are developed in Appendix B] and yield

$$
\begin{aligned}
& h(\mathbf{x}, \mathbf{y}, t)=\frac{N(N-1)}{V^{2}} e^{-\beta_{p} t} \\
& +\beta \frac{N}{V} \int_{0}^{t} d t^{\prime} e^{-\beta_{p} t^{\prime}} \mathcal{G}\left(\mathbf{x}, \mathbf{y}, 2 t^{\prime}\right)
\end{aligned}
$$

when imposing the initial uniform source $Q=1 / V$. The quantity $\beta_{p}$ is a shorthand for $\beta_{p}=\beta /(N-1)$. When branching events are absent $(\beta=0), h(\mathbf{x}, \mathbf{y}, t) \rightarrow h_{i d}(\mathbf{x}, \mathbf{y}, t)$, since spatial correlations are suppressed. The integral of the Green's function appearing in Eq. 18 is bounded thanks to the exponential term, and at long times the correlation function converges to an asymptotic shape. The same asymptotic behaviour is expected 390 for arbitrary initial sources.

\subsection{Relating the spatial moments to $\rho$ and $h$}

The spatial moments of the neutron population defined above can be formally expressed in terms of the particle density $\rho(\mathbf{x}, t)$ and of the correlation function $h(\mathbf{x}, \mathbf{y}, t)$. In particular, for the mean square displacement we have

$$
\left\langle r^{2}\right\rangle(t)=\int|\mathbf{x}|^{2} \rho(\mathbf{x}, t) d \mathbf{x} .
$$

As for the mean square distance between pairs of particles, we have

$$
\left\langle r_{p}^{2}\right\rangle(t)=\frac{\int d \mathbf{x} \int d \mathbf{y}|\mathbf{x}-\mathbf{y}|^{2} h(\mathbf{x}, \mathbf{y}, t)}{\int d \mathbf{x} \int d \mathbf{y} h(\mathbf{x}, \mathbf{y}, t)},
$$

which is to be compared to the ideal average square distance of an uncorrelated population uniformly distributed in the viable volume, namely,

$$
\left\langle r_{p}^{2}\right\rangle_{i d}=\frac{1}{V^{2}} \int d \mathbf{x} \int d \mathbf{y}|\mathbf{x}-\mathbf{y}|^{2}=\frac{1}{2} V^{\frac{2}{3}} .
$$

Deviations of $\left\langle r_{p}^{2}\right\rangle(t)$ from the ideal behaviour $\left\langle r_{p}^{2}\right\rangle_{i d}$ allow quantifying the impact of spatial clustering (Meyer et al., 1996, de Mulatier et al. 2015).

\subsection{Analysis of the homogeneous reactor}

Let us now consider the case of the homogeneous cube reactor introduced above, with size $L$ and $V=L^{3}$. At the boundaries, we impose reflecting (Neumann) conditions. The Green's function for this system reads (Grebenkov, 2013)

$$
\mathcal{G}\left(\mathbf{x}, \mathbf{x}_{0}, t\right)=\sum_{i, j, k}^{\infty} \varphi_{i}(u) \varphi_{i}^{\dagger}\left(u_{0}\right) \varphi_{j}(v) \varphi_{j}^{\dagger}\left(v_{0}\right) \varphi_{k}(w) \varphi_{k}^{\dagger}\left(w_{0}\right) e^{-\alpha_{i, j, k} t}
$$

where

$$
\varphi_{q}(\zeta)=\cos \left(\frac{q \pi \zeta}{L}\right)
$$

are the eigen-modes of the Laplace operator and

$$
\alpha_{i, j, k}=D\left(\frac{\pi}{L}\right)^{2}\left(i^{2}+j^{2}+k^{2}\right)
$$

are the associated eigenvalues. The vector $\mathbf{x}$ is defined by its components, namely, $\mathbf{x}=\{u, v, w\}$. By inspection, the mixing time of the neutron population is identified with $\tau_{D} \propto L^{2} /\left(\pi^{2} D\right)$. The functions $\varphi_{q}^{\dagger}(\zeta)$ are found by imposing ortho-normalization of the eigenmodes, which yields

$$
\varphi_{q}^{\dagger}(\zeta)=\frac{1}{L} \cos \left(\frac{q \pi \zeta}{L}\right)
$$

for $q=0$, and

$$
\varphi_{q}^{\dagger}(\zeta)=\frac{2}{L} \cos \left(\frac{q \pi \zeta}{L}\right)
$$

for $q \geq 1$.

Assuming a uniform spatial distribution $Q=1 / L^{3}$ at time $t=0$, the average density simply reads

$$
\langle n(\mathbf{x}, t)\rangle=\frac{N}{L^{3}} .
$$

The mean square displacement can be easily computed, and yields

$$
\left\langle r^{2}\right\rangle(t)=\int|\mathbf{x}|^{2} \rho(\mathbf{x}, t) d \mathbf{x}=\frac{L^{2}}{4} .
$$

From Eq. 18 we get the pair correlation function

$$
\begin{aligned}
& h(\mathbf{x}, \mathbf{y}, t)=\frac{N(N-1)}{L^{6}} \\
& +\beta N \sum_{i^{\prime}, j^{\prime}, k^{\prime}}^{\infty} \varphi_{i}(u) \varphi_{i}^{\dagger}(q) \varphi_{j}(v) \varphi_{j}^{\dagger}(r) \varphi_{k}(w) \varphi_{k}^{\dagger}(s) \frac{1-e^{-\left(2 \alpha_{i, j, k}+\beta_{p}\right) t}}{2 \alpha_{i, j, k}+\beta_{p}}
\end{aligned}
$$


where the sum is extended to all indexes $i \geq 0, j \geq 0, k \geq 0$, except $i=j=k=0$, and we have set $\mathbf{x}=\{u, v, w\}$ and $\mathbf{y}=$ $\{p, r, s\}$. The series appearing at the right-hand side is bounded, and for times $t \gg \tau_{D}$ we obtain the asymptotic shape of the pair correlation function

$$
\begin{aligned}
& h_{\infty}(\mathbf{x}, \mathbf{y})=\frac{N(N-1)}{L^{6}} \\
& +\beta N \sum_{i^{\prime}, j^{\prime}, k^{\prime}}^{\infty} \frac{\varphi_{i}(u) \varphi_{i}^{\dagger}(q) \varphi_{j}(v) \varphi_{j}^{\dagger}(r) \varphi_{k}(w) \varphi_{k}^{\dagger}(s)}{2 \alpha_{i, j, k}+\beta_{p}} .
\end{aligned}
$$

As for the average square distance, at time $t=0$ we have $\left\langle r_{p}^{2}\right\rangle(0)=\left\langle r_{p}^{2}\right\rangle_{i d}$, as expected. The asymptotic behaviour of $\left\langle r_{p}^{2}\right\rangle(t)$ at times $t \gg \tau_{D}$ can be computed exactly based on Eqs. (28) and 20, , and reads

$$
\begin{aligned}
& \left\langle r_{p}^{2}\right\rangle_{\infty}=\lim _{t \rightarrow \infty}\left\langle r_{p}^{2}\right\rangle(t) \\
& =12 \frac{D}{\beta_{p}}\left[1-\sqrt{\frac{8 D}{\beta_{p} L^{2}}} \tanh \left(\sqrt{\frac{\beta_{p} L^{2}}{8 D}}\right)\right] .
\end{aligned}
$$

By recalling the definitions of the mixing time $\tau_{D}$ and the renewal time $\tau_{R}$, we can rewrite Eq. (29) as

$$
\left\langle r_{p}^{2}\right\rangle_{\infty}=\frac{12 L^{2}}{\pi^{2}} \frac{\tau_{R}}{\tau_{D}}\left[1-\sqrt{\frac{8}{\pi^{2}} \frac{\tau_{R}}{\tau_{D}}} \tanh \left(\sqrt{\frac{\pi^{2}}{8} \frac{\tau_{D}}{\tau_{R}}}\right)\right]
$$

which shows that the impact of the spatial correlations is ruled by the dimensionless ratio between the renewal time and the mixing time. Intuitively, we expect the effects of the correlations to be stronger when the typical time scale of fission renewal is in competition with diffusive mixing (i.e., $\left.\tau_{R} \simeq \tau_{D}\right)_{{ }_{425}}$ and to be weaker when diffusive mixing is faster than renewal (i.e., $\tau_{D} \ll \tau_{R}$ ).

Let us first consider the case of a system where the effects of the spatial correlations induced by clustering are very weak (i.e., $\tau_{R} \rightarrow \infty$ ), which is obtained for a very large number of ${ }_{430}$ particles or a vanishing fission rate. By taking the limit of $\beta \rightarrow$ 0 or equivalently $N \rightarrow \infty$, we have

$$
\left\langle r_{p}^{2}\right\rangle \rightarrow \frac{L^{2}}{2}=\left\langle r_{p}^{2}\right\rangle_{i d}
$$

and we recover the ideal case corresponding to uncorrelated trajectories. In this case, the center of mass of the population obeys

$$
\left\langle r_{c o m}^{2}\right\rangle_{i d}=\left\langle r^{2}\right\rangle-\frac{1}{2} \frac{N-1}{N}\left\langle r_{p}^{2}\right\rangle_{i d}=\frac{L^{2}}{4 N}=\frac{1}{N}\left\langle r^{2}\right\rangle_{i d},
$$

which basically means that for a collection of independent particles the mean square displacement of the center of mass is equal to the mean square displacement of a single particle of the collection, divided by the number of particles.

Consider now a finite reproduction rate $\beta$ and a large but finite number of particles $N \gg 1$. In this case, we can expand Eq. (30) for $\tau_{D} \ll \tau_{R}$, which yields

$$
\begin{aligned}
& \left\langle r_{p}^{2}\right\rangle_{\infty} \simeq \frac{L^{2}}{2}\left[1-\frac{\pi^{2}}{20} \frac{\tau_{D}}{\tau_{R}}+\cdots\right] \\
& =\left\langle r_{p}^{2}\right\rangle_{i d}\left[1-\frac{1}{20} \frac{L^{2}}{N \mathcal{M}^{2}}+\cdots\right],
\end{aligned}
$$

where we have used the definition of the migration area $\mathcal{M}^{2}=$ $D / \beta$. This result relates the typical inter-particle distance to the physical parameters of the reactor model, namely, $N, L$, and $\mathcal{M}^{2}$, and implies in particular that $\left\langle r_{p}^{2}\right\rangle_{\infty}$ will be smaller than in the uncorrelated case because of the effects of spatial clustering.

As for the center of mass, we finally get

$$
\begin{aligned}
& \left\langle r_{\text {com }}^{2}\right\rangle_{\infty}=\left\langle r^{2}\right\rangle-\frac{1}{2} \frac{N-1}{N}\left\langle r_{p}^{2}\right\rangle_{\infty} \simeq \frac{L^{2}}{4 N}\left[1+\frac{\pi^{2}}{20} N \frac{\tau_{D}}{\tau_{R}}+\cdots\right] \\
& =\left\langle r_{c o m}^{2}\right\rangle_{i d}\left[1+\frac{1}{20} \frac{L^{2}}{\mathcal{M}^{2}}+\cdots\right],
\end{aligned}
$$

which again relates the mean square displacement of the center of mass to the physical parameters $N, L$, and $\mathcal{M}^{2}$. In particular, $\left\langle r_{\text {com }}^{2}\right\rangle_{\infty}$ will be larger than that of an uncorrelated system. The correction factor increases for increasing system size $L$, and decreases for increasing migration area $\mathcal{M}^{2}$, as expected on physical grounds.

\subsection{Numerical findings for the homogeneous reactor}

It is interesting to compare the numerical values of $\left\langle r_{\text {com }}^{2}\right\rangle_{\infty}$ predicted by Eq. (34) to the behaviour of the center of mass displayed in Fig. 6 (bottom) and Fig. 7 (bottom). In particular, $\sigma_{\text {com }}=\sqrt{\left\langle r_{\text {com }}^{2}\right\rangle_{\infty}}$ intuitively represents the typical amplitude of the fluctuations of the center of mass around the average. The value of $\mathcal{M}^{2}$ can be estimated by computing $\ell^{2}$ in each Monte Carlo simulation and then setting $\mathcal{M}^{2}=\ell^{2} / 6$ according to diffusion theory. Because of the geometrical symmetries of the cube configuration, along a given axis, say $x$, we get $\sigma_{\text {com }}^{x}=$ $\sqrt{\left\langle r_{\text {com }}^{2}\right\rangle_{\infty} / 3}$.

When the number of particles is kept fixed at $N=10^{4}$, and the size $L$ varies, from Eq. (34) we get for $\sigma_{\text {com }}^{x}$ the values reported in Tab. 1. These findings are entirely consistent with the typical size of the fluctuations of the $x$ component of the center of mass observed in Fig. 6 (bottom): for comparison, the standard deviation $\hat{\sigma}_{c o m}^{x}$ of the recorded statistical series is also reported in Tab. 1 .

\begin{tabular}{|c|c|c|c|}
\hline $\mathrm{L}[\mathrm{cm}]$ & $\ell^{2}\left[\mathrm{~cm}^{2}\right]$ & $\sigma_{c o m}^{x}[\mathrm{~cm}]$ & $\hat{\sigma}_{c o m}^{x}[\mathrm{~cm}]$ \\
\hline \hline 100 & 174 & 1.2 & 1.5 \\
200 & 185 & 4.7 & 5.9 \\
400 & 192 & 18.3 & 19.9 \\
\hline
\end{tabular}

Table 1: Measured and predicted values for the fluctuations of the $x$ component of the center of mass for the homogeneous cube reactor. Here the number of neutrons per generation is kept fixed at $N=10^{4}$, and the reactor size $L$ varies. 


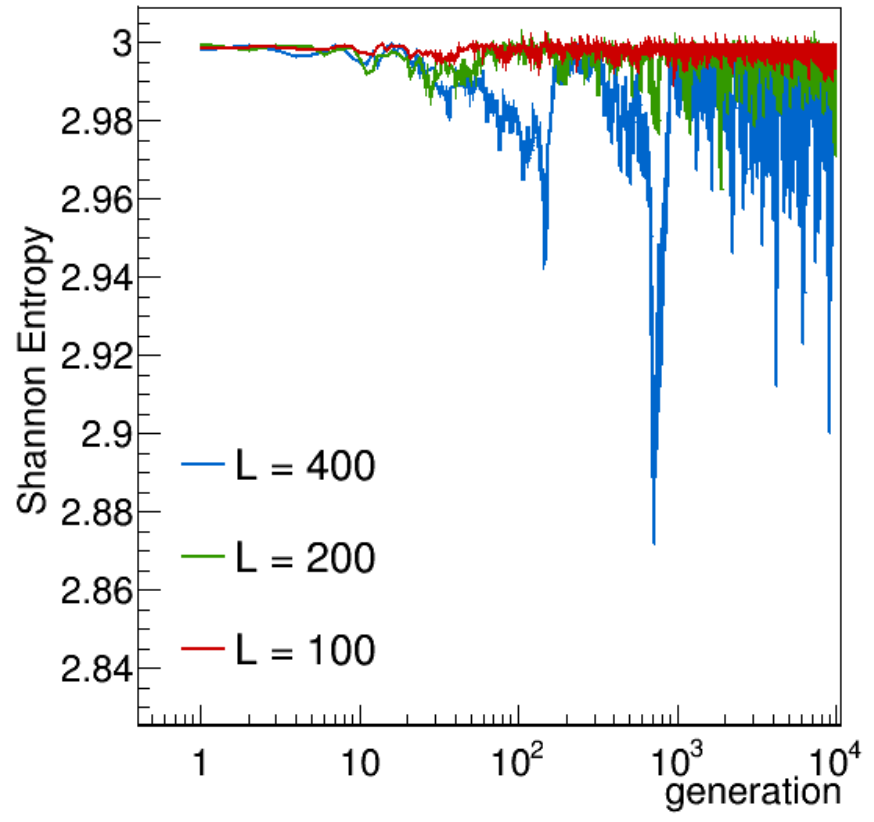

Figure 8: The fuel rod. The behaviour of the measured Shannon entropy $\mathcal{S}(g)$ during Monte Carlo power iteration as a function of generations $g$, for three different fuel rod lengths $L$ and fixed number $N$ of neutrons per generation. The guess source at $g=0$ consists of $N=10^{4}$ neutrons uniformly distributed along the fuel rod. Power iteration is run for $2 \times 10^{4}$ generations. Upper red curve: $L=100 \mathrm{~cm}$; central green curve: $L=200 \mathrm{~cm}$; lower blue curve: $L=400 \mathrm{~cm}$. The mesh chosen for the entropy computation is fixed to 8 identical meshes along the total length of the fuel rod. The theoretical value of the entropy given by Eq. (5) reads $\mathcal{S}_{\infty}=3.0$.

When the reactor size is kept fixed at $L=400 \mathrm{~cm}\left(\ell^{2} \simeq 192\right.$ $\mathrm{cm}^{2}$ ) and $N$ varies, from Eq. (34) we get the $\sigma_{\text {com }}^{x}$ values re- ${ }^{445}$ ported in Tab. 2. Comparison with Fig. 7 (bottom) shows that these predictions are again entirely consistent with the typical size of the fluctuations of the $x$ component of the center of mass observed in our Monte Carlo simulations, whose standard deviation $\hat{\sigma}_{\text {com }}^{x}$ is also reported in Tab. 2

\begin{tabular}{|c|c|c|}
\hline $\mathrm{N}$ & $\sigma_{\text {com }}^{x}[\mathrm{~cm}]$ & $\hat{\sigma}_{\text {com }}^{x}[\mathrm{~cm}]$ \\
\hline \hline $10^{5}$ & 5.8 & 7.3 \\
$10^{4}$ & 18.3 & 19.9 \\
$10^{3}$ & 57.8 & 64.4 \\
\hline
\end{tabular}

Table 2: Measured and predicted values for the fluctuations of the $x$ component of the center of mass for the homogeneous cube reactor. Here the reactor size $L$ is kept fixed at $L=400$ $\mathrm{cm}$, and the number $N$ of neutrons per generation varies.

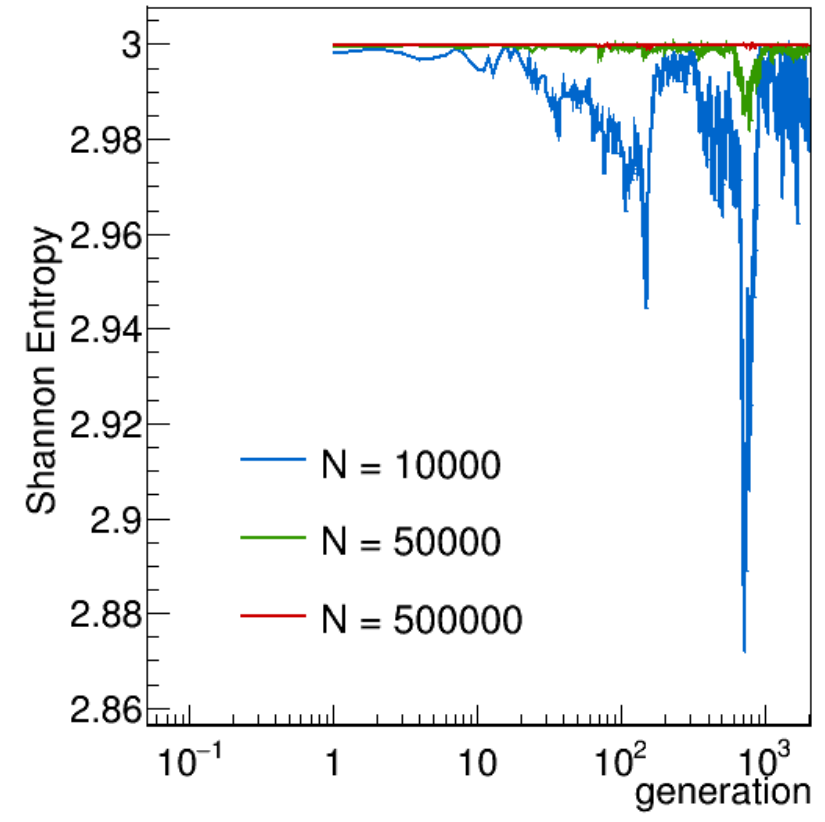

Figure 9: The fuel rod. The behaviour of the measured Shannon entropy $\mathcal{S}(g)$ during Monte Carlo power iteration as a function of generations $g$, for a fixed fuel cell length $L=400 \mathrm{~cm}$ and different numbers $N$ of neutrons per generation. The guess source at $g=0$ consists of $N$ neutrons uniformly distributed along the fuel pin. Power iteration is run for $8 \times 10^{3}$ generations. Upper red curve: $N=5 \times 10^{5}$; central green curve: $N=5 \times 10^{4}$; lower blue curve: $N=10^{4}$. The mesh chosen for the entropy computation is fixed to 8 identical meshes along the total length of the fuel rod. The theoretical value of the entropy given by Eq. (5) reads $\mathcal{S}_{\infty}=3.0$.

\section{Application to realistic reactor configurations}

In the previous Sections, we have applied our analysis to a simplified reactor model. In the following, we would like to ascertain whether the conclusions that were drawn in the case of space- and energy-independent neutron transport actually carry over to more realistic configurations. To this aim, we will examine the behaviour of Monte Carlo power iteration for a fuel rod and for a full reactor core. Monte Carlo simulations have been performed by resorting to the reference code TRIPOLI- $4^{\circledR}$, developed at CEA (Brun et al., 2014, TriPOLI-4 Project Team, 2008).

\subsection{A fuel rod}

Let us begin by considering a fuel rod. This configuration is composed of $\mathrm{UO}_{2}$ fuel at $3.25 \%$ enrichment, with radius 0.407 $\mathrm{cm}$. The fuel pellets are enclosed in a Zircaloy cladding of outer radius $0.477 \mathrm{~cm}$, and a water moderator surrounds the cladding. All materials are kept at $300 \mathrm{~K}$. Reflective boundary conditions have been applied on the system, so that the expected equilibrium distribution for the flux is axially flat in space. The chosen nuclear data library is ENDF/B-VII. 0 . 

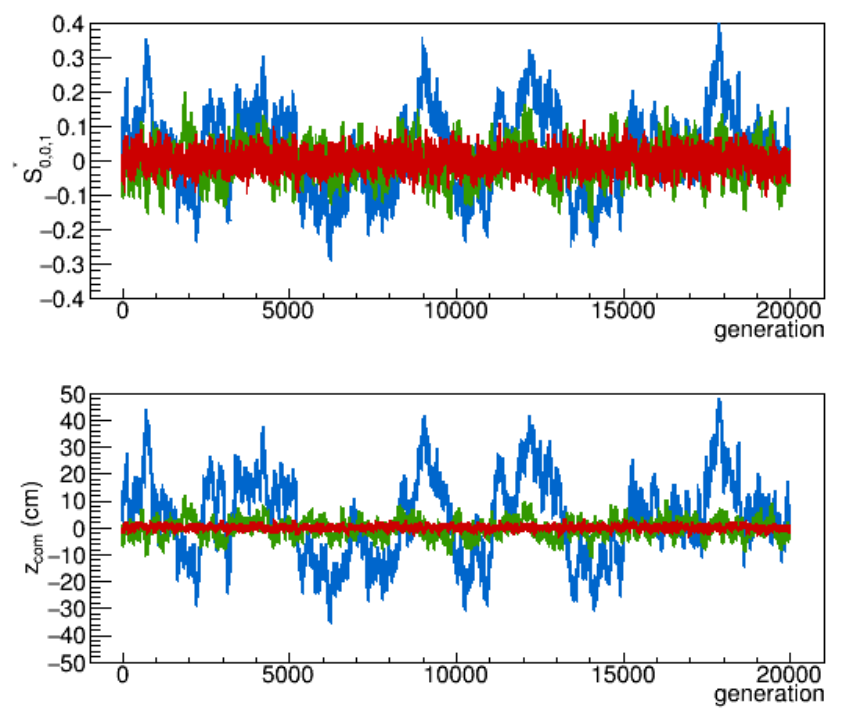

Figure 10: The fuel rod. Monte Carlo power iteration as a function of generations $g$, for three different reactor sizes $L$ and fixed number $N$ of neutrons per generation. The guess source at $g=0$ consists of $N=10^{4}$ neutrons uniformly distributed along the fuel rod. Power iteration is run for $2 \times 10^{4}$ generations. Top. The behaviour of the generalized Shannon entropy $\mathcal{S}_{0,0,1}^{*}(g)$. Red curve: $L=100 \mathrm{~cm}$; Green curve: $L=200 \mathrm{~cm}$; Blue curve: $L=400 \mathrm{~cm}$. Bottom. The behaviour of the center of mass $z_{\text {com }}(\mathrm{g})$. Red curve: $L=100 \mathrm{~cm}$; Green curve: $L=200$ cm; Blue curve: $L=400 \mathrm{~cm}$.

The measured entropy function $\mathcal{S}(g)$ for the fuel rod is shown ${ }^{490}$ in Fig. 8 for fixed number $N$ of simulated particles per generation and varying fuel rod sizes $L$, and in Fig. 9 for fixed $L$ and varying $N$, respectively. When $L$ is varied and $N=10^{4}$, the entropy function converges to a stationary value after about $m=L^{2} / \ell^{2} \simeq 100$ generations for $L=100 \mathrm{~cm}\left(\ell^{2} \simeq 100 \mathrm{~cm}^{2}\right)$, $m=L^{2} / \ell^{2} \simeq 370$ generations for $L=200 \mathrm{~cm}\left(\ell^{2} \simeq 108 \mathrm{~cm}^{2}\right)$, and $m=L^{2} / \ell^{2} \simeq 1400$ generations for $L=400 \mathrm{~cm}\left(\ell^{2} \simeq 112\right.$ $\left.\mathrm{cm}^{2}\right)$. Our predictions are consistent with the numerical findings for $\mathcal{S}(g)$. The entropy at convergence depends on the number $N$ of simulated particles per generations, and an asymptotic $\propto 1 / N$ scaling.

The behaviour of $\mathcal{S}_{0,0,1}^{*}(g)$ for the fuel rod with fixed $N$ fixed and varying $L$ is illustrated in Fig. 10 (top), $z$ being the 495 axis aligned along the rod. As $L$ increases, the fluctuations of $\mathcal{S}_{0,0,1}^{*}(g)$ become stronger. A similar behaviour is found when decreasing the number $N$ of simulated neutrons per generations at fixed reactor size $L$, as shown in Fig. 11 (top): the fluctuations increase by decreasing $N$. The analysis of the center of 500 mass estimator for the fuel rod with fixed $N$ and varying $L$ is illustrated in Fig. 10 (bottom), where we display $z_{\text {com }}(g)$ : when $L$ is small, $z_{\text {com }}(g)$ fluctuates around the symmetry center $z=0$, and the fluctuations are rather mild. As $L$ increases, the evolution of $z_{c o m}(g)$ becomes increasingly erratic. The qualitative behaviour of $z_{\text {com }}(g)$ is similar to that of $\mathcal{S}_{0,0,1}^{*}(g)$. A similar be-505
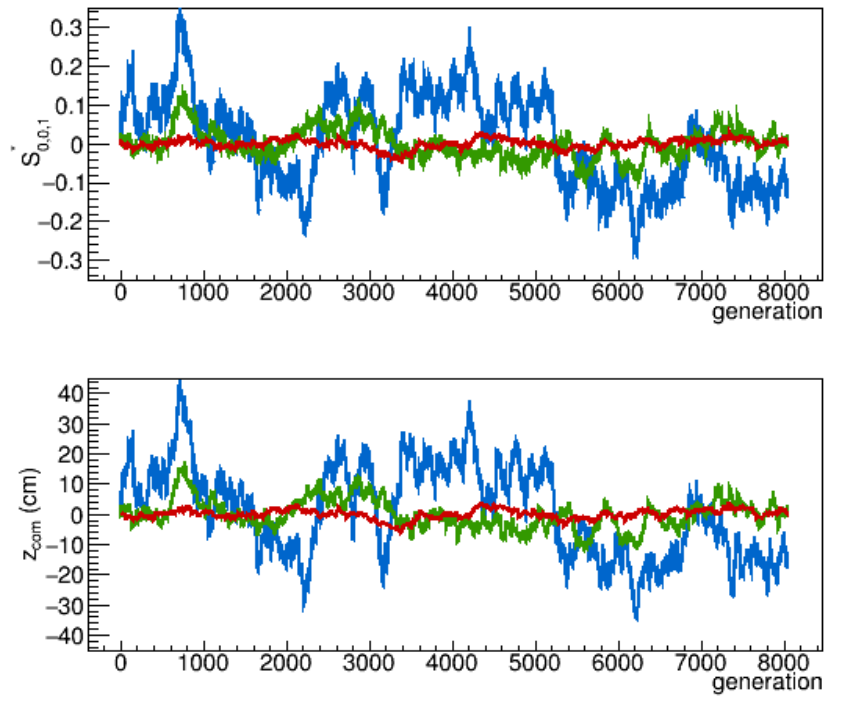

Figure 11: The fuel rod. Monte Carlo power iteration as a function of generations $g$, for a fixed fuel length $L=400 \mathrm{~cm}$ and different numbers $N$ of neutrons per generation. The guess source at $g=0$ consists of $N$ neutrons uniformly distributed along the fuel rod. Power iteration is run for $8 \times 10^{3}$ generations. Top. The behaviour of the generalized Shannon entropy $\mathcal{S}_{0,0,1}^{*}(g)$. Red curve: $N=5 \times 10^{5}$; Green curve: $N=5 \times 10^{4}$; Blue curve: $N=10^{4}$. Bottom. The behaviour of the center of mass $z_{\text {com }}(g)$. Red curve: $N=5 \times 10^{5}$; Green curve: $N=5 \times 10^{4}$; Blue curve: $N=10^{4}$.

haviour is found for fixed $L$ and varying $N$, as shown in Fig. 11 (bottom).

In order to relate the numerical simulation results for $z_{\text {com }}(g)$ to the theory developed above, we have solved Eq. (18) for a one-dimensional homogeneous rod geometry, where neutrons are allowed to only move back and forth along a line of size $L$. Neumann boundary conditions are applied to the ends of the segment. The rod model equations yield

$$
\left\langle z_{\text {com }}^{2}\right\rangle_{\infty}=\left\langle z_{\text {com }}^{2}\right\rangle_{\text {id }}\left[1+\frac{1}{20} \frac{L^{2}}{\mathcal{M}^{2}}+\cdots\right],
$$

with $\left\langle z_{\text {com }}^{2}\right\rangle_{\text {id }}=L^{2} /(12 N)$ and $\mathcal{M}^{2}=\ell^{2} / 2$. For the case of fixed $N=10^{4}$ and varying $L$, the rod model formula yields the $\sigma_{\text {com }}^{z}$ values reported in Tab. 3. Comparison with Fig. 10 (bottom) shows that these predictions are in good agreement with the standard deviation $\hat{\sigma}_{\text {com }}^{z}$ of the recorded statistical series, which is also reported in Tab. 3

When $L=400 \mathrm{~cm}$ and $N$ is varied, Eq. 35 yields the $\sigma_{c o m}^{z}$ values reported in Tab. 4. These predictions are again in good agreement with the standard deviation $\hat{\sigma}_{\text {com }}^{z}$ of the recorded statistical series shown in Fig. 11 (bottom), which is also reported in Tab. 4

\subsection{The Hoogenboom-Martin benchmark}

Let us now turn our attention to a full-scale reactor core model, namely, the Hoogenboom-Martin benchmark (Hoogen- 


\begin{tabular}{|c|c|c|c|}
\hline $\mathrm{L}[\mathrm{cm}]$ & $\ell^{2}\left[\mathrm{~cm}^{2}\right]$ & $\sigma_{\text {com }}^{z}[\mathrm{~cm}]$ & $\hat{\sigma}_{\text {com }}^{z}[\mathrm{~cm}]$ \\
\hline \hline 100 & 100 & 1 & 0.9 \\
200 & 108 & 2.6 & 3.3 \\
400 & 112 & 13.8 & 15.4 \\
\hline
\end{tabular}

Table 3: Measured and predicted values for the fluctuations of the $z$ component of the center of mass for the fuel rod. Here the number of neutrons per generation is kept fixed at $N=10^{4}$, and the fuel rod size $L$ varies.

\begin{tabular}{|c|c|c|}
\hline $\mathrm{N}$ & $\sigma_{\text {com }}^{z}[\mathrm{~cm}]$ & $\hat{\sigma}_{\text {com }}^{z}[\mathrm{~cm}]$ \\
\hline \hline $10^{5}$ & 2 & 1.7 \\
$5 \times 10^{4}$ & 6.2 & 4.9 \\
$10^{4}$ & 13.8 & 15.4 \\
\hline
\end{tabular}

Table 4: Measured and predicted values for the fluctuations of the $z$ component of the center of mass for the fuel rod. Here the fuel rod size size $L$ is kept fixed at $L=400 \mathrm{~cm}$, and the number $N$ of neutrons per generation varies.

boom and Martin, 2009). This benchmark considers a simplified PWR core made of 241 identical fuel assemblies. The fuel composition is representative of a typical depleted core configuration. Two moderator zones are used so as to model the decreasing bottom to top coolant density. We will focus on the cold zero power core configuration (CZP: all materials are at room temperature, $300 \mathrm{~K}$ ), and the chosen nuclear data library is ENDF/B-VII. 0 (Chadwick et al. 2006).

By analogy with the simulations that were carried out for the the evolution of the fission site distribution as a function of the number of generations, for different values $N$ of simulated neutrons per generation. The results for an axial cut at mid-plane are displayed in Fig. 12. We have chosen a uniform neutron source at $g=0$. When $N=10^{5}$, the population evolves toward the fundamental eigenmode, and the fluctuations around the average density are rather mild. When $N$ decreases to $N=10^{4}$, the impact of spatial correlations becomes stronger, and for even smaller $N=5 \times 10^{3}$ neutrons are clearly clustered. Apart from geometeffects due to the shape of the reactor core, the behaviour of the Monte Carlo power iteration of the Hoogenboom-Martin reactor model is not entirely dissimilar to that of the homogeneous cube.

The corresponding entropy function $\mathcal{S}(g)$ is shown in Fig. 13

The mean square displacement per generation has been estimated within the Monte Carlo power iteration and reads $\ell^{2} \simeq$ $356 \mathrm{~cm}^{2}$. Similarly as in the case of the homogeneous cube reactor, the entropy function converges to a stationary value after about $m=H^{2} / \ell^{2} \simeq 380$ generations, where we have used a characteristic reactor size $H \simeq 366 \mathrm{~cm}$ corresponding to the active fuel length (and approximately to the core diameter). The entropy at convergence depends on the number $N$ of simulated particles per generations, and an asymptotic value $\mathcal{S}_{N}$ is eventually reached in the limit of large $N$, with a $\propto 1 / N$ scaling.
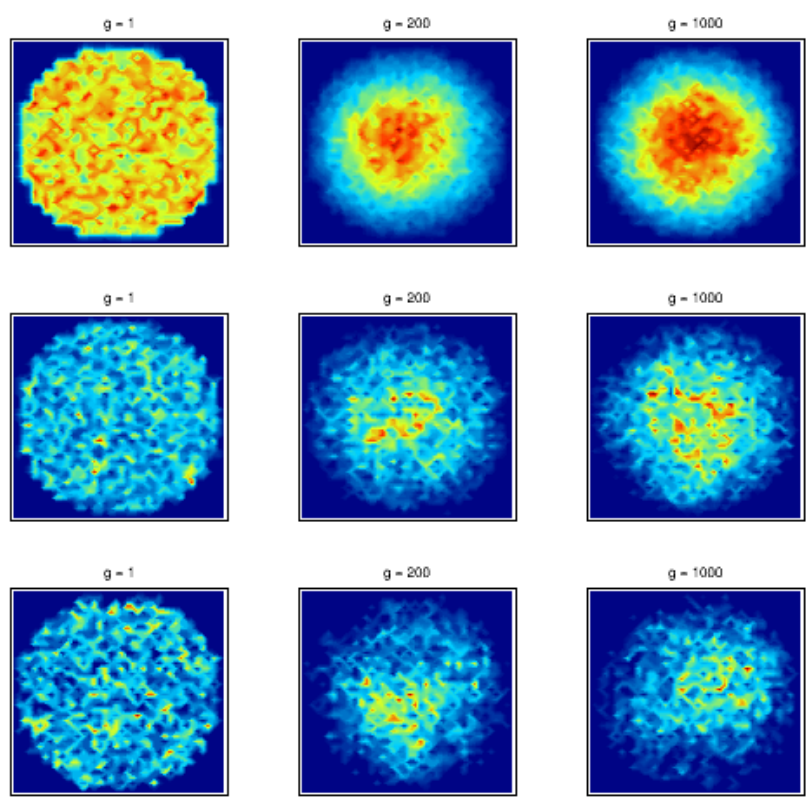

Figure 12: The Hoogenboom-Martin benchmark. Distribution of fission sites during Monte Carlo power iteration as a function of generations $g$, for three different initial population sizes $N$. The guess source at $g=0$ consists of $N$ neutrons uniformly distributed across the reactor. Power iteration is run for $12 \times 10^{4}$ generations. Top: $N=10^{5}$; center: $N=10^{4}$; bottom: $N=$ $5 \times 10^{3}$.

\begin{tabular}{|c|c|c|}
\hline $\mathrm{N}$ & $\sigma_{\text {com }}^{z}[\mathrm{~cm}]$ & $\hat{\sigma}_{c o m}^{z}[\mathrm{~cm}]$ \\
\hline \hline $10^{5}$ & 3.5 & 1.8 \\
$5 \times 10^{4}$ & 5 & 2.2 \\
$10^{4}$ & 11.3 & 5.8 \\
$5 \times 10^{3}$ & 15.9 & 8.6 \\
\hline
\end{tabular}

Table 5: Measured and predicted values for the fluctuations of the $z$ component of the center of mass for the HoogenboomMartin benchmark. The number $N$ of neutrons per generation varies.

The behaviour of the spatial moments of the entropy function and of the center of mass for the Hoogenboom-Martin benchmark are displayed in Fig. 14 (top) and (bottom), respectively. When $N$ is large, the $\mathcal{S}_{0,0,1}^{*}(g)$ is close to zero, and the fluctuations increase by decreasing $N$. Similar results are found for the $x$ and $y$ axis, although geometrical effects come into play due to the cylindrical symmetry. The fluctuations of $z_{\text {com }}(g)$ also increase by decreasing $N$, and the qualitative evolution of $z_{\text {com }}(g)$ is very close to that of $\mathcal{S}_{0,0,1}^{*}(g)$. Equation 18 can be developed for a homogeneous cylinder with Neumann boundaries, which conceptually corresponds to assuming that the water reflector surrounding the reactor core acts as a perfect mirror for neutrons. In this case, for the $z$ component of the center of mass 


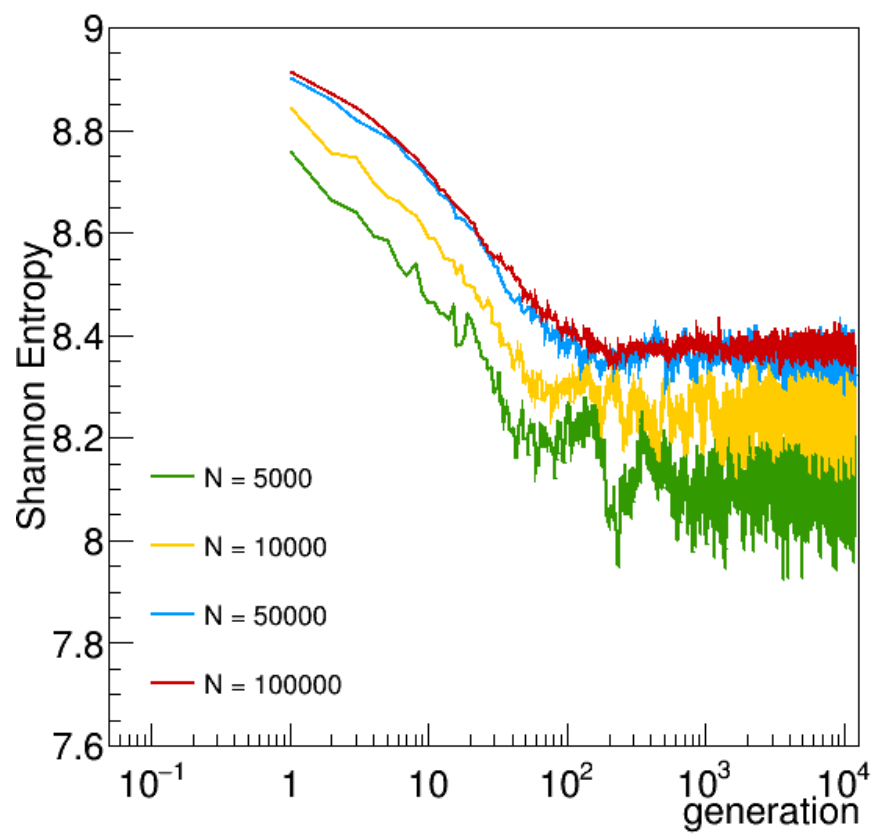

Figure 13: The Hoogenboom-Martin benchmark. The behaviour of the measured Shannon entropy $\mathcal{S}(g)$ during Monte Carlo power iteration as a function of generations $g$, for different initial population sizes $N$. The guess source at $g=0$ consists of $N$ neutrons uniformly distributed in the core. Power iteration is run for $12 \times 10^{4}$ generations. Upper red curve: $N=10^{5}$; central green curve: $N=10^{4}$; lower blue curve: $N=5 \times 10^{3}$.

fluctuations we would get

$$
\left\langle z_{\text {com }}^{2}\right\rangle_{\infty}=\left\langle z_{\text {com }}^{2}\right\rangle_{\text {id }}\left[1+\frac{1}{20} \frac{H^{2}}{\mathcal{M}^{2}}+\cdots\right],
$$

with $\left\langle z_{c o m}^{2}\right\rangle_{i d}=H^{2} /(12 N)$. Numerical estimates for $\sigma_{c o m}^{z}$ are reported in Tab. 5, where we used $\mathcal{M}^{2}=\ell^{2} / 6 \simeq 60 \mathrm{~cm}^{2}$. Com-565 parison with Fig. 14 (bottom) shows that these predictions systematically overestimate (roughly by a factor of 2) the standard deviation $\hat{\sigma}_{\text {com }}^{z}$ of the recorded statistical series, which is also reported in Tab. 5

Deviations of the theoretical formula in Eq. (34) from the $\mathrm{e}_{570}$ observed behaviour of the fluctuations are mostly due to the approximation of entirely neglecting the effects of leakages in our simple model. Nonetheless, the order of magnitude of the fluctuation amplitude and the $1 / \sqrt{N}$ scaling are correctly captured by Eq. 34.

\section{Conclusions}

The statistical behaviour of the neutrons in Monte Carlo power iteration has been analyzed within the framework of 580 branching stochastic processes. In particular, we have shown that it is possible to relate the spatial distributions of neutrons to the key parameters of the simulated configuration, namely, the number of neutrons per generation, the system size, and the
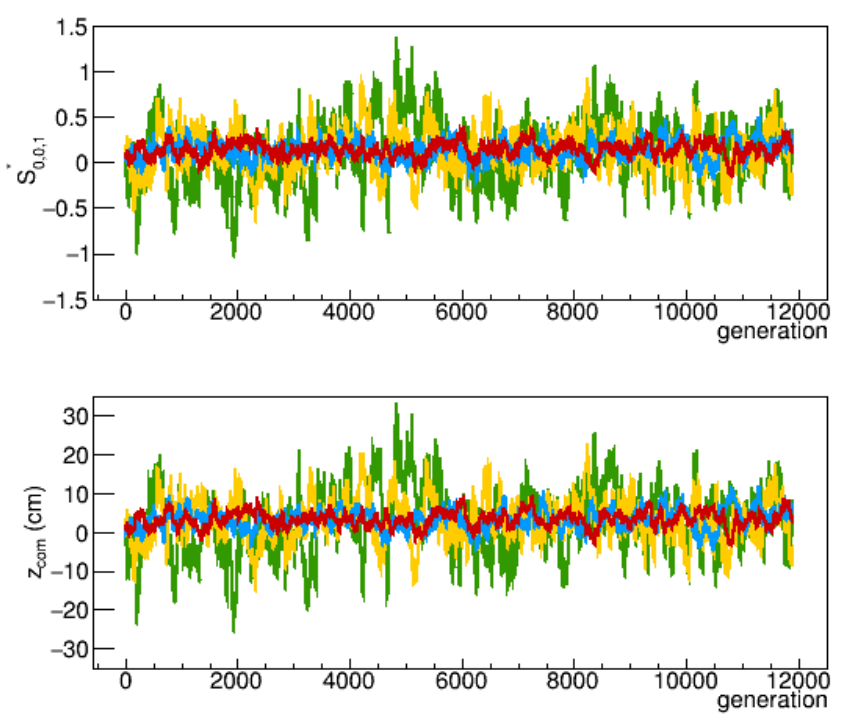

Figure 14: The Hoogenboom-Martin benchmark. Monte Carlo power iteration as a function of generations $g$, for three different initial population sizes $N$. The guess source at $g=0$ consists of $N$ neutrons located at the center of the cube. Power iteration is run for $12 \times 10^{4}$ generations. Top. The behaviour of the generalized Shannon entropy $\mathcal{S}_{0,0,1}^{*}(g)$. Red curve: $N=10^{5}$; blue curve: $N=5 \times 10^{4}$; yellow curve: $N=10^{4}$; green curve: $N=5 \times 10^{3}$. Bottom. The behaviour of the center of mass $z_{\text {com }}(\mathrm{g})$. Red curve: $N=10^{5}$; blue curve: $N=5 \times 10^{4}$; yellow curve: $N=10^{4}$; green curve: $N=5 \times 10^{3}$.

migration area. By resorting to a simple homogeneous cube reactor model, we have illustrated some possible shortcomings of the entropy function in detecting the convergence of the neutron population to equilibrium. We have supported our investigation by analysing the higher moments of the entropy and the center of mass of the population, whose behaviour is directly related to the spatial fluctuations of the neutron population.

A deeper understanding of the spatial behaviour of the neutron population during Monte Carlo power iteration has been achieved by resorting to the theory of branching processes, which allows formally relating the spatial moments (the square pair distance and the square center of mass) to the system parameters. The developed formalism is amenable to exact results (within the diffusion approximation) that are in fairly good agreement with the behaviour observed in the homogeneous cube model.

We have also tested our theoretical findings on more realistic reactor configurations, including a full reactor core and a fuel rod. For the former, we have shown that the formulas derived for a homogeneous cylindrical reactor with reflecting boundaries can capture the scaling of the center of mass fluctuations with respect to the number of particles per generation, although our predictions globally overestimate the fluctuation amplitude. We conjecture that this discrepancy is due to the approximation of having assumed reflecting boundaries: more sophisticated formulas allowing for arbitrary boundary condi- 
would be needed, and this issue will be the subject of future research work. Finally, in the case of the fuel rod (with reflective boundaries) our simplified model is actually capable of correctly assessing the amplitude of the center of mass fluctuations and their scaling with respect to the number of simulated particles per generation and to the fuel rod length.

\section{Acknowledgments}

E. D. , M. N. and A. Z. thank AREVA and Electricité de France (EDF) for partial financial support. J. M. , B. F. and K. S. thank the Consortium for Advanced Simulation of Light Water Reactors (CASL), an Energy Innovation Hub for Modeling and Simulation of Nuclear Reactors under U.S. Department of Energy Contract No. DE-AC05-00OR22725, for providing partial financial support.

\section{Appendix A. Expectation of the Shannon Entropy}

We develop the calculations for the expected Shannon Entropy $\mathcal{S}_{N}$ as defined in Eq. (1). Assume that there are $B$ tally regions in the system, with $N$ identical neutrons. The probability for each neutron to be found at region $i$ is $P_{i}$. After each simulation, $P_{i}$ is estimated as

$$
\widehat{P}_{i}=\frac{k_{i}}{N}
$$

where $k_{i}$ is the number of neutrons in region $i$. Therefore, for the expected entropy we have

$$
\begin{aligned}
& \mathcal{S}_{N}=-\mathbb{E}\left[\sum_{i=1}^{B} \widehat{P}_{i} \log _{2}\left(\widehat{P}_{i}\right)\right] \\
& =\log _{2}(N)-\frac{1}{N} \sum_{i=1}^{B} \mathbb{E}\left[k_{i} \log _{2}\left(k_{i}\right)\right] .
\end{aligned}
$$

For each region $i, k_{i}$ represents the number of occurrences of a neutron fallen in this region. As such, it can be expressed as $a_{620}$ sum of $N$ binomial random variables. Therefore, we obtain

$$
\mathbb{E}\left[k_{i} \log _{2}\left(k_{i}\right)\right]=\sum_{k_{i}=0}^{N}\left(\begin{array}{l}
N \\
k_{i}
\end{array}\right)\left(1-P_{i}\right)^{N-k_{i}} P_{i}^{k_{i}} k_{i} \log _{2}\left(k_{i}\right) .
$$

For the homogeneous reactor model considered in Section 2 , we simply have $P_{i}=1 / B$ and $\mathcal{S}_{N}$ reduces to

$$
\mathcal{S}_{N}=\log _{2}(N)-\frac{B}{N B^{N}} \sum_{k_{i}=0}^{N}\left(\begin{array}{l}
N \\
k_{i}
\end{array}\right)(B-1)^{N-k_{i}} k_{i} \log _{2}\left(k_{i}\right) .
$$

\section{Appendix B. The pair correlation function}

For the sake of completeness, we briefly report here the argument developed in (de Mulatier et al. 2015) for the derivation ${ }^{640}$ of the pair correlation function $h$. Suppose that a given time $t$ we choose a pair of (distinct) neutrons located at positions $\mathbf{x}$ and $\mathbf{y}$, respectively. We would like to determine whether these particle come from a common ancestor, i.e., a fission event occurred at a time $0<t^{\prime}<t$ in the past, in which case the two neutrons are correlated. In our model, we have iposed that the number of individuals in the population is exactly preserved. Then, the fraction of new paris of individuals that can be produced during a time interval $\left(t^{\prime}, t^{\prime}+d t\right)$ is given by $\beta d t /(N-1)$ (Meyer et al. 1996).

The probability for two selected particles not to share a common ancestor in the past is $e^{-\beta_{p} t}$. It follows thus that the probability density for the occurrance of an ancestor at time $t^{\prime}$ is

$$
\psi_{t}\left(t^{\prime}\right)=\beta_{p} e^{-\beta_{p}\left(t-t^{\prime}\right)}
$$

when the observation time is $t$. We have denoted $\beta_{p}=\beta /(N-1)$. It is convenient to split $h$ into the contributions of uncorrelated and correlated events, namely, $h=h^{(1)}+h^{(2)}$. For the former, by construction we simply have $h^{(1)}(\mathbf{x}, \mathbf{y}, t)=e^{-\beta_{p} t} h_{i d}(\mathbf{x}, \mathbf{y}, t)$, which is precisely the contribution of uncorrelated neutrons. As for the correlated events, it can be shown that we have (de Mulatier et al. 2015)

$$
\begin{aligned}
& h^{(2)}(\mathbf{x}, \mathbf{y}, t)=N(N-1) \int_{0}^{t} d t^{\prime} \int_{V} d \mathbf{x}^{\prime} \mathcal{G}\left(\mathbf{x}, \mathbf{x}^{\prime}, t-t^{\prime}\right) \times \\
& \mathcal{G}\left(\mathbf{y}, \mathbf{x}^{\prime}, t-t^{\prime}\right) \psi_{t}\left(t^{\prime}\right) \rho\left(\mathbf{x}^{\prime}, t^{\prime}\right) .
\end{aligned}
$$

The pair correlation function $h$ finally yields

$$
h(\mathbf{x}, \mathbf{y}, t)=\frac{N(N-1)}{V^{2}} e^{-\beta_{p} t}+\beta \frac{N}{V} \int_{0}^{t} d t^{\prime} e^{-\beta_{p} t^{\prime}} \mathcal{G}\left(\mathbf{x}, \mathbf{y}, 2 t^{\prime}\right)
$$

when imposing $Q=1 / V$.

\section{References}

Athreya, K.B., Ney, P., 1972. Branching processes. Grundlehren Series, Springer-Verlag, New York.

Bell, G.I., Glasstone, S., 1970. Nuclear reactor theory, Van Nostrand Reinhold Company, New York.

Brown, F., 2005. LA-UR-05-4983, Los Alamos National Laboratory.

Brown, F., 2009. Proceedings of M\&C2009, Saratoga Springs, N.Y. USA.

Brun, E., et al., 2015. Ann. Nucl. Energy 82, 151-160.

Chadwick, M.B., (2006). Nuc. Data Sheets 107, 2931-3060.

Cover, T.M., Thomas, J.A., 1991. Elements of information theory, John Wiley \& Sons, New York.

5 Dumonteil, E., Courau, T., 2010. Nuclear Technology 172, 120

Dumonteil, E., Malvagi, F., 2012. Proceedings of ICAP2012, Chicago.

Dumonteil, E., Le Peillet, A., Lee, Y.K., Petit, O., Jouanne, C., Mazzolo, A., 2006. Proceedings of PHYSOR2006, Vancouver.

Dumonteil, E., Malvagi, F., Zoia, A., Mazzolo, A., Artusio, D., Dieudonné, C., de Mulatier, C., 2014. Ann. Nuc. Energy 63, 612-618.

Gelbard E.M., Prael, R., 1990. Prog. Nucl. Energy 24, 237.

Grebenkov, D. S., Nguyen, B. T., 2013. SIAM Review 55 601-667.

Hoogenboom, J.E., Martin, W.R., 2010. In Proocedings of the International Conference on Mathematics, Computational Methods and Reactor Physics (M\&C2009), Saratoga Springs, USA; and Hoogenboom, J.E., Martin, W.R., Petrovic, B., (2010). Benchmark specifications, Revision 1.2.

Houchmandzadeh, B., 2008. Phys. Rev. Lett. 101, 078103.

Jacquet, O., Chajari, R., Bay, X., Nourri, A., Carraro, L., 2000. Proceedings of ICRS2000, Lisbon.

L'Abbate, A., Courau, T., Dumonteil, E., 2007. Proceedings of PHYTRA1, Marrakech.

Li and Vitanyi, 1997. An Introduction to Kolmogorov Complexity and Its Applications, Springer. 
Lux, I., Koblinger, L., 1991. Monte Carlo particle transport methods: Neutron and photon calculations, CRC Press, Boca Raton.

Miao, J., Forget, B. and Smith, K., 2016. Ann. Nuc. Energy 92, 81-95.

Meyer, M., Havlin, S., Bunde, A., 1996. Phys. Rev. E 54, 5567.

de Mulatier, C., Dumonteil, E., Rosso, A., Zoia, A., 2015. J. Stat. Mech. P08021.

650 Pázsit, I., Pál, L., 2008. Neutron Fluctuations: A Treatise on the Physics of Branching Processes, Elsevier, Oxford.

Rief, H., Kschwendt, H., 1967. Nuc. Sci. Eng. 30, 395-418.

Shi, B., Petrovic, B., 2010. In Proceedings of the ANS International Conference on the Physics of Reactors (PHYSOR 2010), Pittsburgh, PA, May 9-14 (2010).

Shi, B., Petrovic, B., 2010. In Proceedings of the Joint International Conference on Supercomputing in Nuclear Applications and Monte Carlo 2010 (SNA+MC2010), Tokyo, Japan, October 17-21, 2010.

Sjenitzer, B.L., Hoogenboom, J.E., 2011. Ann. Nucl. Eng. 38, 2195.

660 T. M. Sutton, 2014. In Proceedings of the SNA+MC2013 conference, Paris, France.

T. M. Sutton, 2014. In Proceedings of the M\&C+SNA+MC2015 conference, Nashville, TN, USA.

Tripoli-4 Project Team, 2008. Tripoli-4 User guide, Rapport CEA-R-6169.

665 Ueki, T., Brown, F., 2003. Proceedings of M\&C2003, Gatlinburg, Tennessee.

Ueki, T., Brown, F., Parsons, D.K., Kornreich, D.E., 2003. Nucl. Sci. Eng. 145, 279

Ueki, T., Brown, F., Parsons, D.K., Warsa, J.S., 2004. Nucl. Sci. Eng. 148, 374.

Ueki, T., 2005. Nucl. Sci. Eng. 151, 283.

670 Ueki, T., 2012. J. Nucl. Sci. Tech. 49, 1134-1143.

Wenner, M., Haghighat, A., 2007. Trans. Am. Nuc. Soc. 97, 647-650.

Wenner, M., Haghighat, A., 2008. In Proceedings of the Physor2008 conference, Interlaken Switzerland.

Williams, M.M.R., 1974. Random Processes in Nuclear Reactors, Pergamon Press, Oxford

Young, W.R., Roberts, A.J., Stuhne, G., 2001. Nature, 412, 328.

Zhang, Yi-C., Serva, M., Polikarpov, M., 1990. J. Stat. Phys. 58, 849-861.

Zoia, A., Dumonteil, E., Mazzolo, A., Mohamed, S., 2012. J. Phys. A: Math Theor. 45, 425002 .

680 Zoia, A., Dumonteil, E., Mazzolo, A., de Mulatier, C., Rosso, A., 2014. Phys. Rev. E 90, 042118. 